\title{
Photometric variability of young brown dwarfs in the $\sigma$ Orionis open cluster
}

\author{
J. A. Caballero ${ }^{1}$, V. J. S. Béjar ${ }^{1}$, R. Rebolo ${ }^{1,2}$, and M. R. Zapatero Osorio ${ }^{3}$ \\ 1 Instituto de Astrofísica de Canarias, 38205 La Laguna, Tenerife, Spain \\ e-mail: zvezda@ll.iac.es \\ 2 Consejo Superior de Investigaciones Científicas, Spain \\ 3 LAEFF - INTA, PO Box 50727, 28080, Madrid, Spain
}

Received 9 January 2004 / Accepted 28 May 2004

\begin{abstract}
We have carried out multi-epoch, time-series differential $I$-band photometry of a large sample of objects in the south-east region of the young ( $\sim 3 \mathrm{Myr})$, nearby ( $\sim 350 \mathrm{pc}) \sigma$ Orionis open cluster. A field of $\sim 1000 \mathrm{arcmin}^{2}$ was monitored during four nights over a period of two years. Using this dataset, we have studied the photometric variability of twenty-eight brown dwarf cluster candidates with masses ranging from the stellar-substellar boundary down to the planetary-mass domain. We have found that about $50 \%$ of the sample show photometric variability on timescales from less than one hour to several days and years. The amplitudes of the $I$-band light curves range from less than 0.01 up to $\sim 0.4$ mag. A correlation between the near-infrared excess in the $K_{\mathrm{s}}$ band, strong $\mathrm{H} \alpha$ emission and large-amplitude photometric variation is observed. We briefly discuss how these results may fit the different scenarios proposed to explain the variability of cool and ultracool dwarfs (i.e. magnetic spots, patchy obscuration by dust clouds, surrounding accretion discs and binarity). Additionally, we have determined tentative rotational periods in the range 3 to $40 \mathrm{~h}$ for three objects with masses around $60 M_{\text {Jup }}$, and the rotational velocity of $14 \pm 4 \mathrm{~km} \mathrm{~s}^{-1}$ for one of them.
\end{abstract}

Key words. stars: low mass, brown dwarfs - Galaxy: open clusters and associations: individual: $\sigma$ Orionis techniques: photometric

\section{Introduction}

The photometric variability of brown dwarfs and very low mass stars close to the substellar limit has received significant attention during the past few years. The studies, mostly performed in the red part of the optical spectrum, using broad-band (Martín \& Zapatero Osorio 1997; Terndrup et al. 1999; Bailer-Jones \& Mundt 1999, 2001; Martín et al. 2001; Gelino et al. 2002; Joergens et al. 2003) and narrow-band filters (Tinney \& Tolley 1999; Clarke et al. 2002a,b), indicate that approximately one third of the late M- and L-type dwarfs show variability with amplitudes from $\sim 10$ to $\sim 80$ mmag. Several of these objects have been reported as periodic variables, with periods in the range from half an hour to ten days. Photometry in the near-infrared region, where cool dwarfs emit most of their output energy, has also been performed in young clusters (Carpenter et al. 2002; Zapatero Osorio et al. 2003 - hereafter ZO03) and in the field (Bailer-Jones \& Lamm 2003; Enoch et al. 2003). The detected amplitudes of variation are in the range $0.05-0.2 \mathrm{mag}$, and tentative periods of $\sim 1.5$ to $3 \mathrm{~h}$ have been reported. On the other hand, time-resolved spectroscopy has emphasized the study of dust/temperature- or activity-sensitive spectral features such as water vapour, $\mathrm{TiO}, \mathrm{FeH}$ and $\mathrm{CrH}$ bands or $\mathrm{H} \alpha$ emission, respectively (Nakajima et al. 2000; Hall 2002; Bailer-Jones 2002; Burgasser et al. 2002; Liebert et al. 2003; Clarke et al. 2003). Among the scenarios proposed to explain the observed levels of photometric and spectroscopic variability, we may list cool and/or hot corotating magnetically-induced spots in the atmospheres, heterogeneous or patchy coverage of photospheric clouds of grains of solid condensates, (sub)stellar discs surrounding the objects, and the presence of very low-mass companions in close orbits, which may produce eclipses or mass-transfer episodes.

Photometric variability studies of objects with confirmed membership in star clusters with known astrophysical properties have advantages to those in the field. Age, metallicity and distance are the same for the sample of cluster members. Because they lie within a well-defined photometric sequence, different luminosities correspond to different masses. Hence, the monitoring of a sample of cluster objects usually leads to the exploration of the properties of members in a wide range of masses. Additionally, wide field cameras can simultaneously observe a relatively large number of sources under the same sky and instrumental conditions. At very young ages (e.g., a few Myr), fast-rotating completely convective brown dwarfs may generate a magnetic field strong enough to 
produce solar-like surface spots or chromospheric activity. Also, accretion discs may not yet have been completely dissipated. Investigation of the rotation of very young brown dwarfs is especially important for understanding crucial phenomena related to substellar formation, such as angular momentum evolution, the relation between disc and central object, and disc evaporation.

Near-infrared and optical wide-field photometric monitoring of stars in the Orion region has already been performed, especially in the area surrounding the Orion Nebula Cluster (Herbst et al. 2000, 2002; Carpenter et al. 2001; Rebull 2001, and references therein). In particular, in the $\sigma$ Orionis region, evidence of variability has been found in a large variety of objects, from the massive magnetic helium-strong B2 Vp star in the central OB multiple stellar system, through solar-like stars, to objects below the stellar-substellar threshold (e.g., Fedorovich 1960; Landstreet \& Borra 1978; Bailer-Jones \& Mundt 2001).

In this paper we present differential photometry of 32 lowmass member candidates of the young $\sigma$ Orionis open cluster, which belongs to the Ori OB1b association. Walraven photometry and HIPPARcos measurements of the distance to the brightest stars of the association point to a distance of $\sim 350 \mathrm{pc}$ (Brown et al. 1994; Perryman et al. 1997; de Zeeuw et al. 1999). Optical extinction towards the $\sigma$ Orionis stellar system and the surrounding region seems to be small $\left(A_{\mathrm{V}}<1\right.$ mag Lee 2003; Béjar et al. 2001; Oliveira et al. 2002). The age of the cluster is estimated between 1 and $8 \mathrm{Myr}$, with $3 \mathrm{Myr}$ being the most likely value (Zapatero Osorio et al. 2002a; Oliveira et al. 2002).

\section{Observations and analysis}

The observations were performed at Observatorio del Roque de Los Muchachos, on La Palma (Canary Islands). We used the four-chip Wide Field Camera (WFC) mosaic, mounted at the prime focus of the $2.5 \mathrm{~m}$ Isaac Newton Telescope. The detector consists of four thinned EEV4280 $2 \mathrm{k} \times 4 \mathrm{k}$ CCDs with a pixel size of $13.5 \mu \mathrm{m}$, corresponding to a pixel scale of $0.333^{\prime \prime}$.

Monitoring of a $\sim 0.29$ degree $^{2}$ area south-east of the centre of the $\sigma$ Orionis cluster was carried out during two epochs: three consecutive nights, from 2000 December 30 through 2001 January 1 (hereafter, WFC00), and on 2003 January 8 (WFC03). Several Landolt standard stars (Landolt 1992) were observed during 2003 January 8, which allowed us to calibrate the WFC03 I magnitude. Thirty-three different exposures, each with completeness magnitude $I \sim 21.5$, resulted from these observations. We used the RGO I filter, which resembles the Johnson-Cousins-Kron I. Although the nights were clear, seeing conditions varied slightly during the runs. In the first epoch, a longer exposure time was used, and the counts of several bright objects lay in the non-linear regime when the seeing improved. In Table 1 is a log of the observations with other useful information. We give the date, number of exposures taken each observing night, effective mean airmass, average full width half maximum $(F W H M)$ in arcsec and exposure time in seconds. Above the horizontal line is shown the WFC00 dataset, and below is the WFC03 dataset. The WFC00 data have
Table 1. Log of the observations.

\begin{tabular}{lcccc}
\hline \hline Date & $\begin{array}{c}\text { Nos. } \\
\text { exposures }\end{array}$ & Airmass & $\begin{array}{c}F W H M \\
{\left[{ }^{\prime}\right]}\end{array}$ & $\begin{array}{c}\text { Exp. time } \\
{[\mathrm{s}]}\end{array}$ \\
\hline 30 Dec. 2000 & 5 & $1.23-1.70$ & $1.4-1.6$ & 1500 \\
31 Dec. 2000 & 10 & $1.17-1.50$ & $0.9-1.4$ & 1500 \\
01 Jan. 2001 & 6 & $1.17-1.58$ & $1.2-1.8$ & 1500 \\
\hline 08 Jan. 2003 & 12 & $1.17-1.81$ & $1.0-2.0$ & 1200 \\
\hline
\end{tabular}

already been used, together with near-infrared observations, to study the photometric variability of the young, low-mass brown dwarf S Ori 45 (ZO03).

The reduction of the images was performed within the IRAF $^{1}$ environment, using standard packages (IMRED-CCDRED). Raw images were bias-subtracted and flat-fielded before alignment. A dither pattern, with displacements of several times the seeing, was used in order to subtract the contribution of the fringing appropriately. To accomplish this, a flat-field image for each night was constructed using bias-subtracted scientific images.

Alignments of the WFC00 and WFC03 images were done separately, because of the presence of a 0.6-arcmin shift of pointing centres between epochs. Residual fringing was below the level of $0.8 \%$ over the CCDs. A study of the average FWHM and sky background of each image was made before calculating the aperture photometry. This analysis was done for each detector individually. We used the IRAF DAOPHOT package to perform the photometric analysis. Aperture photometry was performed using nine different apertures per image: 0.50, 0.60, $0.70,0.80,0.90,1.00,1.25,1.50$, and 2.00 times the average $F W H M$. The inner radius of the sky annulus was 5.00 times the average $F W H M$, while the width of the annulus was fixed at 8 pixels. Instrumental magnitudes for 29514 objects were obtained.

\subsection{Differential photometry}

The data analysis (selection of sources, calculation of the reference magnitudes, statistical analysis, obtaining of light curves, preliminary time-series analysis) was done mainly with the MATLAB $^{2}$ interactive system.

We required the mean of the IRAF magnitude errors from sources of the whole sample to be lower than 0.25 mag for each of the nine apertures. In order to avoid source confusion, especially when faint objects are close to bright ones or in crowded fields, objects with standard deviations of the centroid coordinates larger than 1.5 pixels $\left(\sim 0.5^{\prime \prime}\right)$ were rejected. Taking into account these requirements, sources with counts close to saturation, very faint objects that appear close to very bright stars, cosmic rays and chip defects were mostly discarded.

\footnotetext{
IRAF is distributed by National Optical Astronomy Observatories, which are operated by the Association of Universities for Research in Astronomy, Inc., under cooperative agreement with the National Science Foundation.

2 MATLAB is a high-performance language for technical computing developed by The MathWorks, Inc.
} 
Instrumental magnitudes were transformed into differential magnitudes, $I_{\text {diff }}$, by subtracting a reference value (i.e. a zero-point magnitude level). This reference magnitude for each CCD and exposure was calculated using a large number of bright, non-variable stars located over the entire monitored area. They were chosen using an iterative procedure: only low-photometric-noise sources with standard deviation $\sigma\left(I_{\text {diff }}\right)<0.020 \mathrm{mag}$ (in the WFC00 dataset) and $<0.015 \mathrm{mag}$ (WFC03), and with magnitudes in the range $17.5 \mathrm{mag}<I<$ $21.0 \mathrm{mag}$, were selected from an initial sample of reference star candidates. Each CCD of the WFC00 and WFC03 datasets was treated separately. The final sample of reference objects ( $\sim 100$ per chip) was the intersection between the WFC00 and $\mathrm{WFC} 03$ reference samples. We note that a photometrically stable brown-dwarf target, when compared to other objects of similar magnitude, may also be considered a reference "star" if it matches the criteria given above.

Reference magnitudes were calculated following BailerJones \& Mundt (2001). The method is based in a mean of fluxes of the reference stars, where the brightest sources, with the lowest Poissonian photon-count errors, dominate the final reference magnitude. However, a simple mean of the magnitudes of the reference stars was also worked out, to check for any significant difference. Because of the large size of the sample of reference stars, both zero-point magnitudes were similar. As the standard deviation of the difference between the mean of fluxes and the mean of magnitudes was lower than $1 \mathrm{mmag}$, we used only the mean-of-fluxes reference level in the subsequent calculations.

We studied the dependence of the reference magnitude for each CCD on time, airmass and seeing. No trends were detected, so we did not applied either airmass- or seeingdependent high-order corrections. Computations were made for each Wide Field Camera chip separately, as each CCD has different characteristics (i.e. gain, electronic noise and offsets between instrumental magnitudes and apparent magnitudes). Also, for each chip the average $F W H M$ is different at a given time because of the different distances to the centre of the focal plane.

We performed the previous analysis in the WFC00 dataset for each of the nine apertures ( 0.5 to $2.0 \times F W H M)$ and chose the $0.70 \times F W H M$ aperture, which both minimized the photon contribution of nearby contaminant sources and maximized the signal-to-noise ratio and, as a result, provided the largest number of reference stars.

An additional offset was added to the $I_{\text {diff }}$ values of each object in order to transform them into apparent magnitudes, calibrated using the Landolt standard stars observed during the WFC03 run. As a result, for each object the mean WFC03 $I_{\text {diff }}$, denoted by $\overline{I_{03}}$, is equal to the real $I$-band magnitude.

Finally, multi-epoch calibrated $I$-band light curves were obtained for more than 8000 objects that fulfilled all the criteria given above.

\section{Substellar candidates}

Of the $32 \sigma$ Orionis brown dwarf candidates studied here, 19 were photometrically discovered at optical wavelengths by

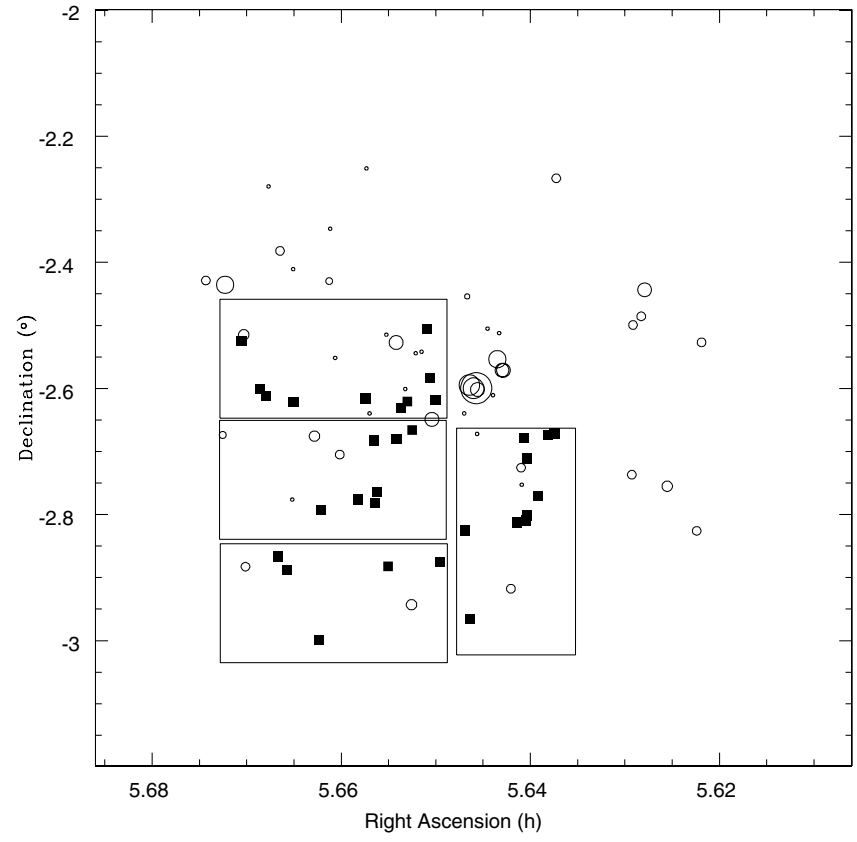

Fig. 1. Spatial location of the 32 brown-dwarf member candidates in the $\sigma$ Orionis cluster studied in this article, shown with filled squares. Stars brighter than $I=15.0 \mathrm{mag}$ that presumably belong to the cluster are represented with open circles. The circle radii are inversely proportional to the magnitude. Rectangles denote the four CCD chips of the Wide Field Camera.

Béjar et al. (1999, 2004). Their near-infrared colours are consistent with membership of the cluster. In addition, many of them have been confirmed spectroscopically. The remaining 13 objects were selected from a combined $I J$ survey conducted with the Wide Field Camera $(I)$ at the Isaac Newton Telescope and the ISAAC instrument $(J)$ at the Very Large Telescope Antu/UT 1. Details of this survey will be given in a forthcoming paper. The 32 selected targets for the present photometric analysis have $I$ magnitudes between 16.0 and 22.5 mag. Neither brighter nor fainter objects yielded reliable differential photometry either because of non-linearity or excessively faint signals in each exposure, respectively. In Fig. 1, we show the spatial location of the targets, stars brighter than $I=15.0 \mathrm{mag}$ that presumably belong to the $\sigma$ Orionis cluster (based on spectral type and/or proper motion determinations) and the position on the sky of the WFC mosaic. We provide in Table 2 the complete sample, together with relevant spectroscopic information from the literature (e.g., spectral types and pseudo-equivalent widths of lithium absorption at $6708 \AA$ and $\mathrm{H} \alpha$ emission).

The spectral types of the targets range from $\sim \mathrm{M} 5.5$ to $\sim \mathrm{L} 2$. According to theoretical models, at the age of the cluster ( $\sim 3 \mathrm{Myr}$ ), these roughly correspond to masses ${ }^{3}$ between $\sim 80$ and $\sim 8 M_{\text {Jup }}$. The upper limit is slightly larger than the theoretical minimum mass for hydrogen fusion, the borderline between stars and brown dwarfs. The lower limit is below the deuterium-burning mass threshold, suggested as the frontier between brown dwarfs and planetary-mass objects (e.g., Saumon et al. 1996). These mass estimates may be affected

\footnotetext{
${ }^{3}$ Conversion between solar and Jupiter masses: $1 M_{\odot}=1047.56 \pm$ $0.08 M_{\text {Jup }}$.
} 
Table 2. Photometric and spectroscopic data for the $32 \sigma$ Orionis cluster members considered in our study.

\begin{tabular}{|c|c|c|c|c|c|c|c|c|}
\hline \multirow[t]{2}{*}{ Name } & \multirow[t]{2}{*}{$I$} & \multirow[t]{2}{*}{$I-J$} & \multirow[t]{2}{*}{ Sp. type } & \multirow[t]{2}{*}{$\overline{p E W(\operatorname{Li} \mathrm{I})(\AA)}$} & \multicolumn{4}{|c|}{$p E W(\mathrm{H} \alpha)(\AA)$} \\
\hline & & & & & BZOR & $\mathrm{ZO}$ & ByN & $\mathrm{M}$ \\
\hline S Ori J054000.2-025159 & $16.21 \pm 0.04$ & $2.15 \pm 0.05$ & - & - & - & - & - & - \\
\hline S Ori J053902.1-023501 & $16.41 \pm 0.03$ & $1.62 \pm 0.05$ & - & - & - & - & - & - \\
\hline S Ori $16^{a}$ & $16.62 \pm 0.03$ & $1.94 \pm 0.04$ & - & - & - & - & - & - \\
\hline S Ori J053847.2-025756 & $16.75 \pm 0.02$ & $2.23 \pm 0.03$ & - & - & - & - & - & - \\
\hline S Ori J053829.0-024847 & $16.81 \pm 0.02$ & $1.80 \pm 0.06$ & M $6.0 \pm 0.5$ & - & - & - & $<5$ & \\
\hline S Ori J053954.3-023719 & $16.82 \pm 0.03$ & $1.94 \pm 0.04$ & - & - & - & - & - & - \\
\hline S Ori J053825.4-024241 ${ }^{a}$ & $16.86 \pm 0.02$ & $1.73 \pm 0.03$ & - & - & - & - & - & - \\
\hline S Ori $25^{a}$ & $16.93 \pm 0.03$ & $2.16 \pm 0.03$ & M $7.5 \pm 0.5$ & 0.6 & $45.0 \pm 1.0$ & - & $42 \pm 8$ & $44 \pm 1$ \\
\hline S Ori J053826.1-024041 ${ }^{a}$ & $16.93 \pm 0.02$ & $1.95 \pm 0.04$ & M $8.0 \pm 0.5$ & - & - & - & $4 \pm 2$ & - \\
\hline S Ori $27^{a}$ & $17.03 \pm 0.03$ & $2.15 \pm 0.03$ & M $7.0 \pm 0.5$ & $0.74 \pm 0.09$ & $6.1 \pm 1.0$ & $5.7 \pm 0.5$ & $5 \pm 2$ & - \\
\hline S Ori J053922.2-024552 & $17.05 \pm 0.03$ & $1.65 \pm 0.05$ & - & - & - & - & - & - \\
\hline S Ori J054014.0-023127 & $17.07 \pm 0.03$ & $2.60 \pm 0.04$ & - & - & - & - & - & - \\
\hline S Ori $28^{a}$ & $17.14 \pm 0.03$ & $1.81 \pm 0.03$ & - & - & - & - & - & - \\
\hline S Ori $31^{a}$ & $17.23 \pm 0.02$ & $1.87 \pm 0.07$ & M $7.0 \pm 0.5$ & - & - & - & $2.5 \pm 0.9$ & - \\
\hline S Ori $30^{a}$ & $17.29 \pm 0.03$ & $1.98 \pm 0.03$ & M $6.0 \pm 0.5$ & - & - & - & $16 \pm 6$ & - \\
\hline S Ori $32^{a}$ & $17.34 \pm 0.03$ & $1.85 \pm 0.03$ & - & - & - & - & - & - \\
\hline S Ori J054004.5-023642 ${ }^{a}$ & $17.51 \pm 0.03$ & $2.02 \pm 0.04$ & - & - & - & - & - & - \\
\hline S Ori $36^{a}$ & $17.71 \pm 0.03$ & $2.20 \pm 0.04$ & - & - & - & - & - & - \\
\hline S Ori J053918.1-025257 & $18.64 \pm 0.04$ & $2.51 \pm 0.04$ & - & - & - & - & - & - \\
\hline S Ori $42^{a}$ & $19.04 \pm 0.03$ & $2.48 \pm 0.03$ & M $7.5 \pm 0.5$ & - & - & - & $89 \pm 12$ & - \\
\hline S Ori $45^{a}$ & $19.49 \pm 0.02$ & $2.57 \pm 0.02$ & M $8.5 \pm 0.5$ & $2.4 \pm 1.0$ & $60.0 \pm 1.0$ & $33 \pm 9$ & $26 \pm 15$ & - \\
\hline S Ori J053929.4-024636 & $19.73 \pm 0.03$ & $2.55 \pm 0.03$ & - & - & - & - & - & - \\
\hline S Ori $71^{a}$ & $20.02 \pm 0.03$ & $2.69 \pm 0.04$ & $\mathrm{~L} 0.0 \pm 0.5$ & - & - & - & $700 \pm 80$ & - \\
\hline S Ori J053849.5-024934 & $20.08 \pm 0.02$ & $2.80 \pm 0.04$ & - & - & - & - & - & - \\
\hline S Ori $51^{a}$ & $20.23 \pm 0.03$ & $2.85 \pm 0.07$ & M $9.0 \pm 0.5$ & - & - & - & 25: & - \\
\hline S Ori $50^{a}$ & $20.48 \pm 0.03$ & $2.88 \pm 0.04$ & M $9.0 \pm 0.5$ & - & - & - & $<10$ & - \\
\hline S Ori $47^{a}$ & $20.49 \pm 0.02$ & $3.16 \pm 0.03$ & $\mathrm{~L} 1.5 \pm 1.0$ & $4.3 \pm 0.5$ & - & $\leq 6$ & 25: & - \\
\hline S Ori J053944.5-025959 & $20.74 \pm 0.04$ & $2.72 \pm 0.05$ & - & - & - & - & - & - \\
\hline S Ori $53^{a}$ & $20.96 \pm 0.02$ & $2.87 \pm 0.03$ & M $9.0 \pm 0.5$ & - & - & - & $<10$ & - \\
\hline S Ori J054007.0-023604 & $21.16 \pm 0.03$ & $2.84 \pm 0.04$ & - & - & - & - & - & - \\
\hline S Ori J053956.8-025315 & $21.25 \pm 0.04$ & $2.97 \pm 0.05$ & - & - & - & - & - & - \\
\hline S Ori J053858.6-025228 & $22.17 \pm 0.05$ & $3.54 \pm 0.06$ & - & - & - & - & - & - \\
\hline
\end{tabular}

${ }^{a}$ References: Béjar et al. (1999) and Béjar et al. (2004) (BZOR); Zapatero Osorio et al. (1999, 2002a) (ZO); Barrado y Navascués et al. (2001, 2002, 2003) (ByN); Muzerolle et al. (2003) (M).

by uncertainties of the evolutionary models at very young ages (see the discussion by Baraffe et al. 2002, 2003). The spectrophotometric sequence of the $\sigma$ Orionis cluster in the substellar domain is known from previous studies (references in footnote of Table 2 and Béjar et al. 2001). A colour-magnitude diagram $I$ vs. $I-J$, such as the one shown in Fig. 2, provides a reliable way of determining the membership of candidates without spectroscopic data.

\subsection{Ho emission}

According to the standard scenario of classical T Tauri stars, broad $\mathrm{H} \alpha$ emission lines are caused by the accretion flow of free-falling gas. The infalling gas, in this case, is accreted during the early stages of star formation on to the disc or at the base of high-latitude magnetic accretion columns on the stellar surface (Königl 1991, and references therein). Several authors have suggested the extrapolation of this picture to objects below the stellar/substellar mass limit.

The great majority of the spectroscopically confirmed brown dwarfs show $\mathrm{H} \alpha$ emission (see Table 2), which may be related to chromospheric activity or to the presence of a circum(sub)stellar disc. The asymmetric, extraordinarily strong $\mathrm{H} \alpha$ emission of the cool substellar object S Ori 71 should be noted. Barrado y Navascués et al. (2002) estimated its mass to be between 22 and $13 M_{\text {Jup }}$. They measured a pseudoequivalent width of $700 \pm 80 \AA$, which is among the strongest $\mathrm{H} \alpha$ emissions so far seen in a young late-type dwarf. S Ori 25 , S Ori 42 and S Ori 45 also show strong $\mathrm{H} \alpha$ emission, which is $\sim 10-20$ times greater than the average emission of other $\sigma$ Orionis sources of similar spectral type. According to Barrado y Navascués \& Martín (2003), such strong emission 


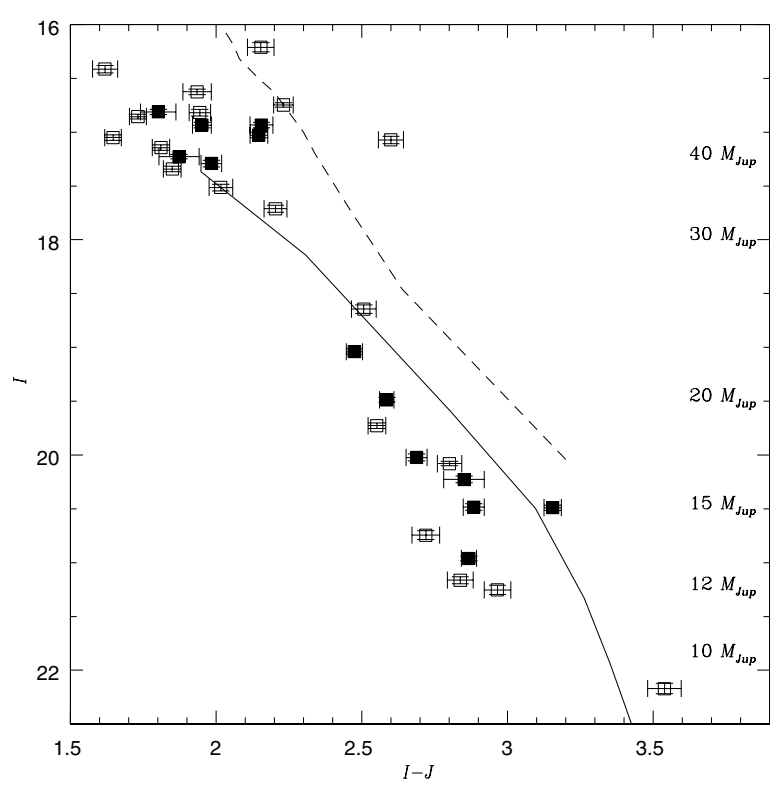

Fig. 2. $I$ vs. $I-J$ colour-magnitude diagram of the 32 brown-dwarf member candidates under study. Spectroscopically confirmed substellar cluster members are shown with filled squares, while open squares indicate objects with no available spectroscopic data. The solid and dashed lines are $3 \mathrm{Myr}$ colour-magnitude isochrones for substellar objects, based on models by the Lyon group (Baraffe et al. 2002; Chabrier et al. 2000, respectively). Approximate masses are provided to the right.

is consistent with disc accretion phenomena. Other cluster objects show weak to moderate $\mathrm{H} \alpha$ emission lines.

Lamm et al. (2004) have recently detected a strong correlation between photometric variability in periodic variables and $\mathrm{H} \alpha$ emission in a sample of pre-main sequence stars in the young (2-4 Myr) open cluster NGC 2264. However, no evidences for a correlation for the irregular variables have been found.

\subsection{Near-infrared excess}

We have used the 2MASS All-Sky Catalog of Point Sources (Cutri et al. 2003) to study the presence of any near-infrared excesses in our targets that could be an indication of warm surrounding discs. In Fig. 3 is shown the $I-J$ vs. $I-K_{\mathrm{s}}$ diagram for 17 of the brown-dwarf candidates studied, with 2MASS $J H K_{\mathrm{s}}$ photometry more precise than 0.1 mag (i.e. targets brighter than $I=17.5 \mathrm{mag}$, approximately). The solid and dashed lines are the 3 Myr colour-colour isochrones for substellar objects, based on models by the Lyon group (Baraffe et al. 2002; and Chabrier et al. 2000, respectively). All the substellar candidates except two lie in the dashed isochrone within the error bars. The outliers are S Ori 27 and S Ori J053825.4-024241. S Ori 27 is an M $6.5 \pm 0.5$ brown dwarf with an estimated mass of $\sim 40 M_{\text {Jup }}$. According to its $I-J$ colour, S Ori J053825.4-024241 may be an $\sim$ M 6-type brown dwarf for which optical spectroscopy is not available. While S Ori 27 seems to move towards bluer colours, S Ori J053825.4-024241 shows a clear reddening that suggests an excess of infrared emission at $2.2 \mu \mathrm{m}$ or, alternatively, it may

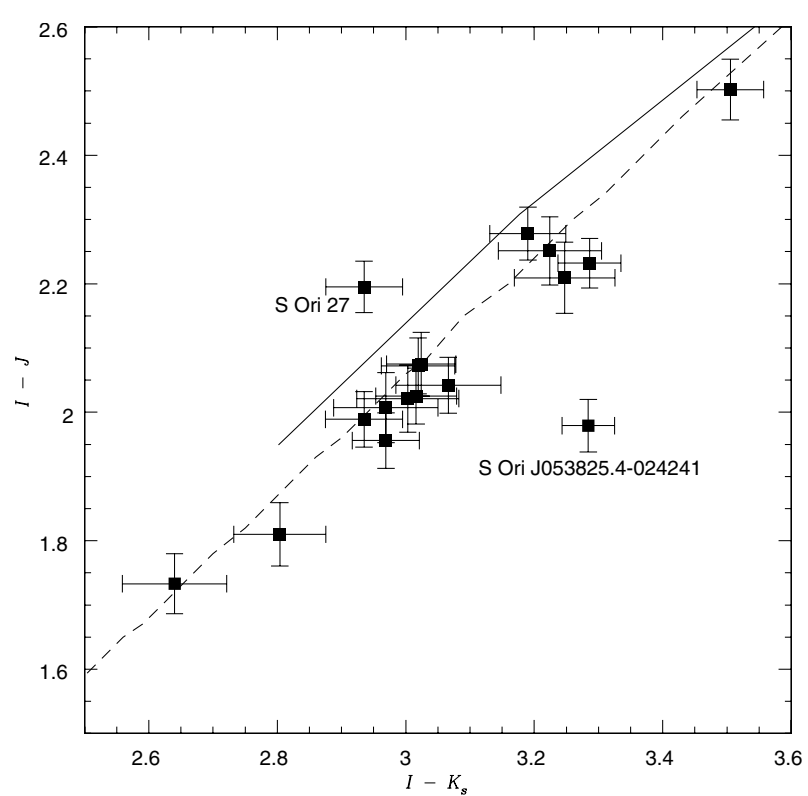

Fig. 3. $I-J$ vs. $I-K_{\mathrm{s}}$ colour-colour diagram. All the brown-dwarf candidates studied with 2 MASS $J H K_{\mathrm{s}}$ photometry data with precision better than 0.1 mag are shown. The isochrones are described in the caption of Fig. 2.

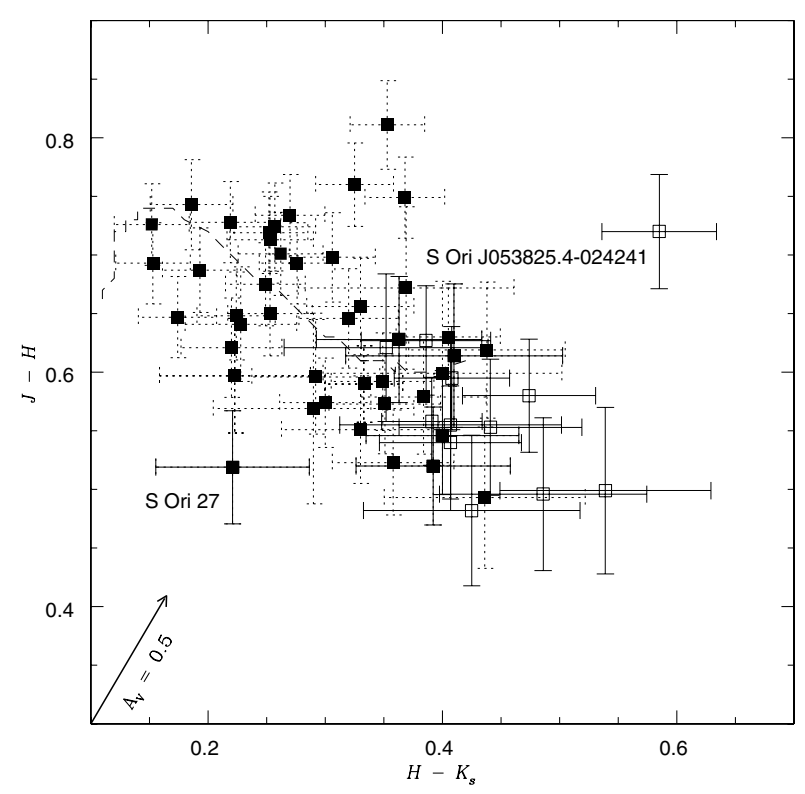

Fig. 4. $J-H$ vs. $H-K_{\mathrm{s}}$ colour-colour diagram. Small filled squares with dotted error bars are for spectroscopically confirmed objects of the $\sigma$ Orionis cluster. Open squares with solid error bars denote the objects studied in this paper. Spectroscopically confirmed objects are plotted as filled squares. The dashed line is the $3 \mathrm{Myr}$ colour-colour isochrone for substellar objects, based on Chabrier et al. (2000) models. Late-K and early-M stars are located in the upper left corner of the figure, while the mid- and late-M dwarfs are close to the center.

be affected by interstellar extinction. These objects do not lie close to the cluster sequence in the $J-H$ vs. $H-K_{\mathrm{s}}$ diagram, as shown in Fig. 4 where, again, only $2 \mathrm{MASS} J H K_{\mathrm{s}}$ data more precise than 0.1 mag have been plotted.

Recently, Oliveira et al. (2003) have obtained $L^{\prime}$ photometry of $\sigma$ Orionis stars with late $\mathrm{K}$ and early $\mathrm{M}$ spectral types, 
concluding that about $50 \%$ of the stellar cluster population show a considerable infrared excess at $3.8 \mu \mathrm{m}$. About half of the $\sigma$ Orionis stars harbour dusty envelopes that may occur in the form of discs. We note that the frequency of strong $\mathrm{H} \alpha$ emissions in $\sigma$ Orionis stars and brown-dwarf members (Zapatero Osorio et al. 2002a,b; Barrado y Navascués 2003; Muzerolle et al. 2003) is not as high as the frequency of $L^{\prime}$-band infrared excesses. Furthermore, the rate of $K$-band infrared excesses is rather low as compared to the $L^{\prime}$-band (Oliveira et al. 2002). Mid-infrared excesses are better disc indicators.

Evidence for accretion discs, dusty envelopes and the existence of a $\mathrm{T}$ Tauri-like phase in young brown dwarfs has been claimed for other similarly young clusters and starforming regions, such as $\rho$ Ophiuchi (Wilking et al. 1999; Testi et al. 2002; Natta et al. 2002), the Trapezium (Muench et al. 2001), Chamaeleon I (Natta \& Testi 2001; Persi et al. 2001; Jayawardhana et al. 2002), R Coronae Australis (Fernández \& Comerón 2001; Barrado y Navascués et al. 2003), IC 348 (Jayawardhana et al. 2003), Lupus 3 (Comerón et al. 2003), and TW Hydrae (Mohanty et al. 2003).

\section{Criteria of variability}

The scatter of the data points in a light curve represents a measure of the photometric variability, or at least sets an upper limit to the variability. The standard deviation, $\sigma(I)$, of the light curve is the most used variability indicator, although other parameters have been also proposed: the peak-to-peak amplitude, the average of the absolute relative magnitudes, the "range" (the difference in median values of the upper 15\% and the lower $15 \%$ of measurements), variability and median statistics, as the $\chi^{2}$ or the $\tilde{\eta}$, and other indices or tests as the Kolmogorov-Smirnov test. Some of the parameters need posterior Monte Carlo simulations to quantify the likelihood of variability. Discussions about some variability indicators can be found in Stetson (1996), Bailer-Jones \& Mundt (1999), Herbst et al. (2002) and Enoch et al. (2003). Here, we will define a few criteria of variability according to different timescales. We will compare the photometry of our $\sigma$ Orionis sample with the data of field sources of similar brightness. For consistency purposes, all of the light curves are treated in the same manner.

We define $\sigma(I)$ as the standard deviation of the light curves for each night. The $\sigma(I)$ vs. $I$ diagram shown in Fig. 7 illustrates the strong dependency of the photometric standard deviation on the target apparent magnitude. The lower envelope of $\sigma(I)$ is roughly flat at the level of $5 \mathrm{mmag}$ in the $I \sim 17.0-19.5 \mathrm{mag}$ range, while it increases exponentially above $\sim 20.5$ mag because of the Poissonian photoncount error. On the other hand, the scatter also increases below $\sim 16.5$ mag because of saturation and non-linear effects in bright sources. We note that to compute the $\sigma(I)$, three $I$ values in the WFC00 epoch and one value in the WFC03 were rejected for all the objects, as the measured point spread functions (PSF) in the corresponding images were far from the average PSF for each epoch.

The three brightest brown dwarfs in the sample of $32 \sigma$ Orionis objects $(I<16.7 \mathrm{mag})$ will not be considered in the discussion of Sect. 5 because a significant fraction of the light curve points was recorded with counts in the non-linear regime of the detector. Likewise, the light curve of S Ori J053858.6-025228, a substellar object fainter than $I=$ $22 \mathrm{mag}$, was discarded because of the large Poissonian error. For the remaining 28 brown dwarfs, what we call the final sample, the photometry is reliable.

The light curves of our final sample are displayed in Figs. 5 and 6. For comparison purposes, we have used a common display: vertical height has been fixed to $0.5 \mathrm{mag}$ and the temporal scale is constant along the horizontal axis. The Modified Julian Date (MJD = JD -2 400000.5 ) is indicated below, and the two epochs are shown. We have not taken into account the heliocentric correction, as the maximum difference between JD and HJD (Heliocentric Julian Date) is of the order of only a few seconds over a period of three nights. The reference star light curves are similar to that of S Ori 36.

\subsection{Short timescales}

In order to find photometric variability on very short timescales (in a series of consecutive exposures, i.e., minutes to hours), we compared the $\sigma(I)$ of the light curves of the targets with those of field stars of similar magnitude. For each target we defined a short-term variability parameter, $\varpi_{\mathrm{ST}}$, given by the following equation:

$\varpi_{\mathrm{ST}}=\frac{N^{-}}{N^{+}+N^{-}}$

where the subscript "ST" stands for short-term, and $N^{+}$and $N^{-}$ are the number of sources in the same magnitude bin with $\sigma(I)$ larger and lower, respectively, than the $\sigma(I)$ of the object under consideration. High values of $\varpi_{\mathrm{ST}}$ imply more likely variability. The width of the bin was fixed to $0.2 \mathrm{mag}$, centred on the I magnitude of the object. Typically about one hundred objects with similar brightness and, hence, with similar photometric error, were considered in the comparison. Such a narrow bin was selected to minimize the error contribution from faint sources and sources in the non-linear domain, which show a larger photometric error that could mask the intrinsic variability of the target.

We will impose a cut at $\varpi_{\mathrm{ST}}=0.95$ to separate variable from non-variable sources. Targets with $\varpi_{\mathrm{ST}} \geq 0.95$ are considered to be very likely short-term variable brown dwarfs in our sample. This criterion demands that for a target brown dwarf to be detected as variable it must be significantly more variable than field objects in the same magnitude bin. Statistically, the light curves of the variable sources display photometric amplitudes that are among the 5\% largest amplitudes for their magnitude bins. This is as if there is only a $5 \%$ chance that a brown dwarf which is not truly variable is incorrectly classified as variable (i.e. 5\% chance of a false positive detection). We note that the distribution of $\varpi_{\mathrm{ST}}$ is rather flat between 0 and 1 within a given magnitude bin. However, the distribution of $\sigma(I)$ is asymmetric: most of the data points fall close to the mean photometric IRAF errors, while only a small percentage lies far from this location (see Fig. 7). 

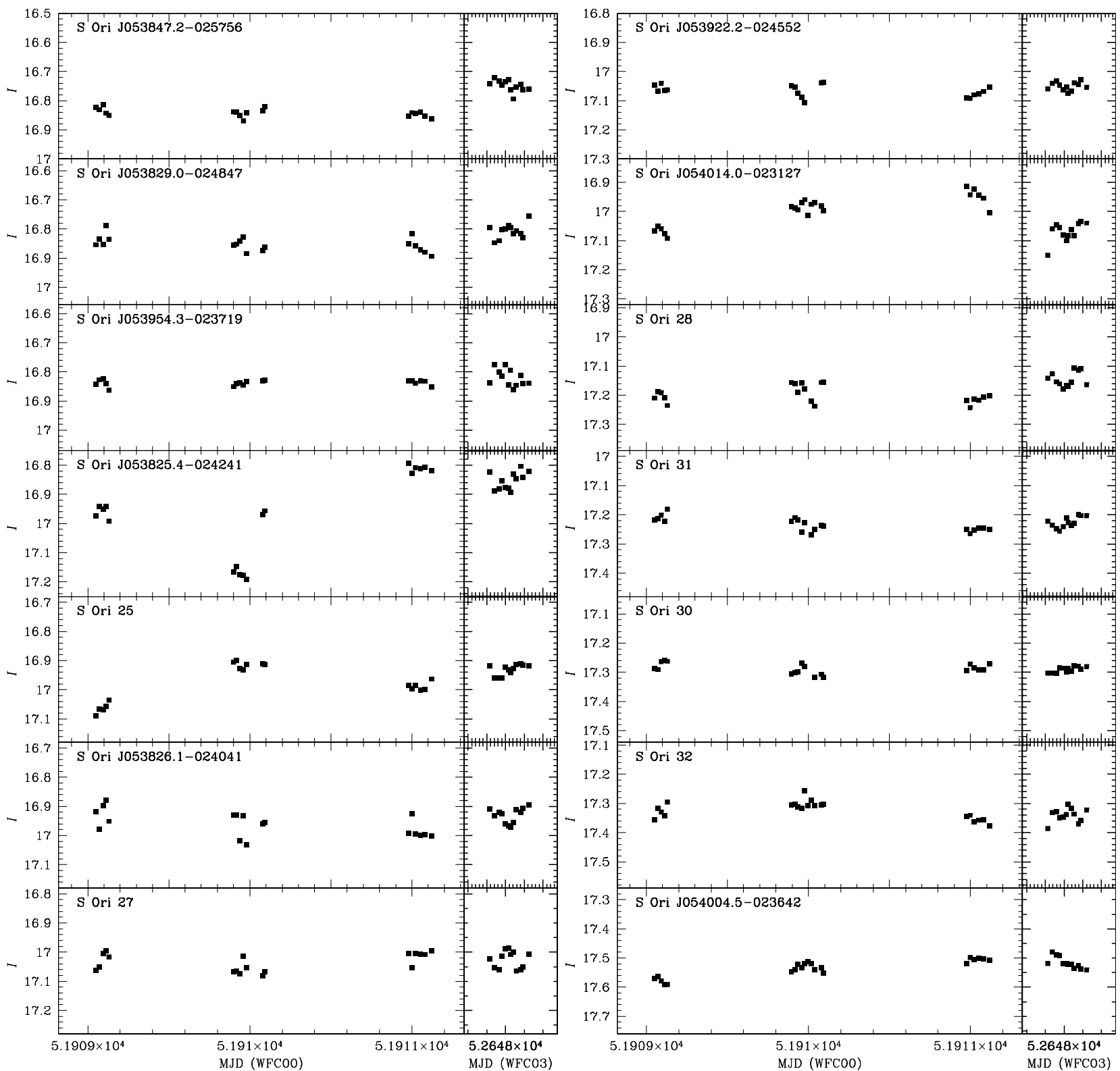

Fig. 5. Light curves of the fourteen brightest objects in the final sample.

\subsection{Intermediate timescales}

To detect variability in a target from one night to another (hereafter mid-term variability, i.e. scale of a few days), we compared the averaged magnitudes (of both targets and field sources), $\bar{I}_{i}$, computed for each night, $i$. We list the mean magnitudes of our sample in Table 3. The comparison is performed by obtaining the absolute value of $\overline{I_{i}}-\overline{I_{j}}$ per object, where $i$ and $j$ represent two different nights. In order to avoid the bias introduced by short-term photometric variability, we have divided the differences by the standard error of the mean magnitudes. Hence, we have defined a dimensionless absolute magnitude difference, $\delta(I)_{i j}$, as:

$\delta(I)_{i j}=\frac{\left|\overline{I_{i}}-\overline{I_{j}}\right|}{\sqrt{\frac{\sigma\left(I_{i}\right)^{2}}{n_{i}}+\frac{\sigma\left(I_{j}\right)^{2}}{n_{j}}}}$ where $n$ stands for the number of photometric data points per night. By using mean $I$-band magnitudes per night we are increasing the sensitivity to mid-term variability.

We have selected likely mid-term variables in our sample in a similar manner as we picked short-term variable brown dwarfs. We have used the definition of Eq. (1), which we now name $\varpi_{\mathrm{MT}}$, where the subscript stands for "mid-term". $N^{+}$ and $N^{-}$represent, in this case, the number of sources in the same magnitude bin with $\delta(I)_{i j}$ larger and lower, respectively, than the $\delta(I)_{i j}$ of the object under consideration. Figure 8 shows the dimensionless differences against apparent magnitudes for the second and third nights of the WFC00 epoch. Similar diagrams are obtained when considering other possible combinations, e.g. first and second nights, second and third nights. We note that mid-term variability can be investigated only in the WFC00 data. As in the previous analysis of short-term variability, the bin magnitude is chosen to be 0.2 mag centred on the 

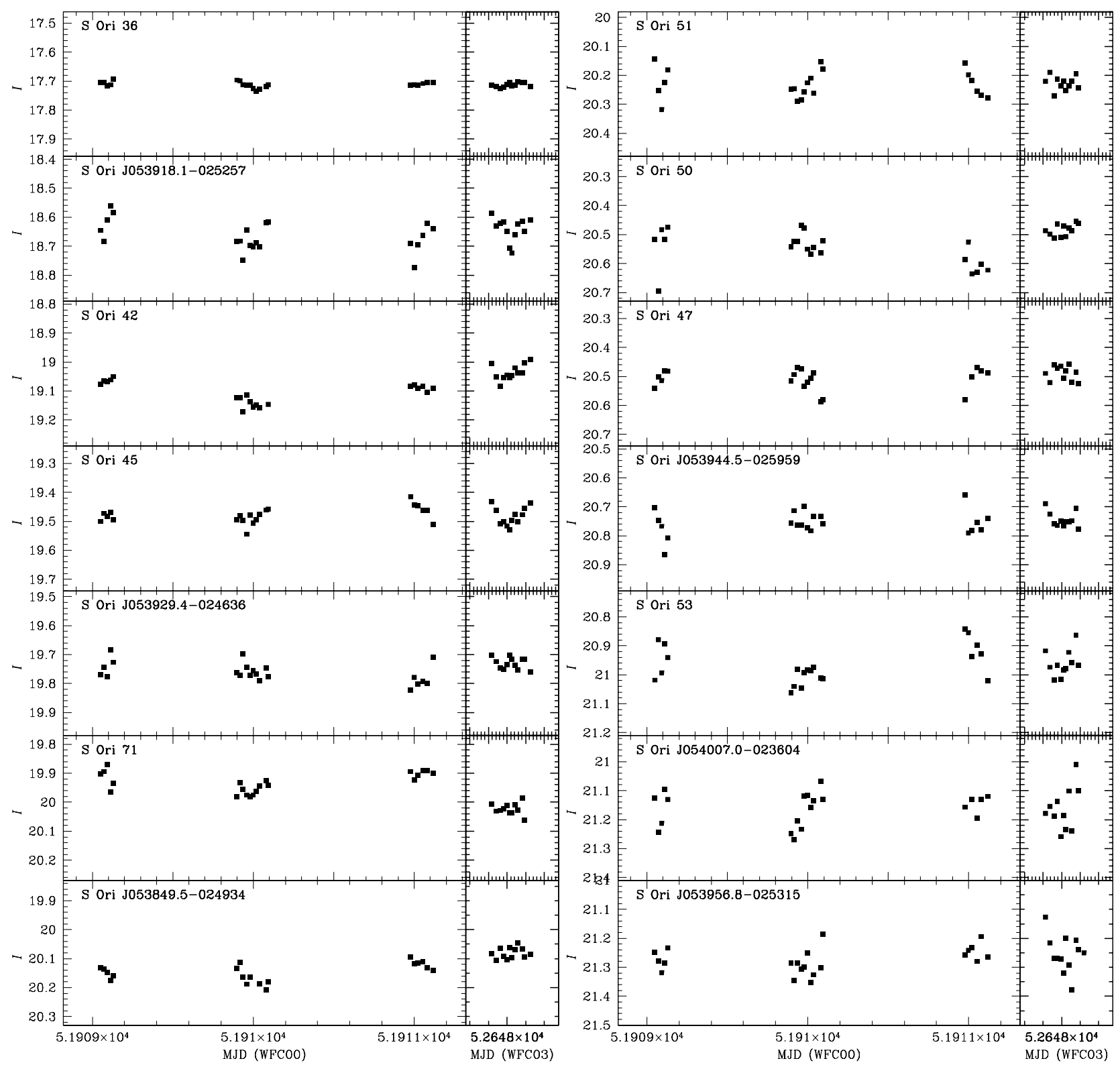

Fig. 6. Light curves of the fourteen faintest objects in the final sample.

I magnitude of each target. According to our criterion, likely mid-term variable brown dwarfs have $\varpi_{\mathrm{MT}} \geq 0.95$.

\subsection{Long timescales}

For long-term variability, which takes into account differences between the first epoch of observations (WFC00) and the second (WFC03), separated by about 2 years, we applied a criterion similar to that of mid-term variability. Now, the only WFC03 night is compared to each of the three WFC00 nights using the dimensionless magnitude differences, $\delta(I)_{i j}$. The parameter that accounts for long-term variability is named $\varpi_{\mathrm{LT}}$.

Long-term variable $\sigma$ Orionis brown dwarfs in our sample have $\varpi_{\mathrm{LT}} \geq 0.95$. The $\varpi_{\mathrm{LT}}$ calculated for the comparison between the first WFC00 night and the WFC03 epoch is depicted as a function of apparent $I$ magnitude in Fig. 9. Similar diagrams are obtained for the remaining two WFC00 nights. We note that according to the adopted criterion of variability, all mid-term variables could also be identified as long-term variable sources. This is so because we have performed comparisons of each WFC00 night to the WFC03 epoch. Mid-term variable sources might be true long-term variables but we cannot discriminate easily with our data. Hence, we have excluded mid-term variables from our analysis of long-term variability.

\section{Discussion}

We provide in Table 4 the variability parameters obtained for the three different timescales and for our target sample.

\subsection{Long-term variable brown dwarfs}

Two brown dwarfs, namely S Ori J053847.2-025756 and S Ori 42 , vary significantly $\left(\varpi_{\mathrm{LT}} \geq 0.95\right)$ from the WFC00 to 
Table 3. Mean magnitude and standard deviation for the light curves of each night ${ }^{a}$.

\begin{tabular}{|c|c|c|c|c|}
\hline Name & $\overline{I_{00 \# 1}} \pm \sigma$ & $\overline{I_{00 \# 2}} \pm \sigma$ & $\overline{\overline{I_{00 \# 3}} \pm \sigma}$ & $\overline{I_{03}} \pm \sigma$ \\
\hline S Ori J054000.2-025159 & $16.203 \pm 0.012:$ & $16.34 \pm 0.05$ & $16.17 \pm 0.03:$ & $16.21 \pm 0.05$ \\
\hline S Ori J053902.1-023501 & $16.51 \pm 0.03$ & $16.58 \pm 0.04:$ & $16.47 \pm 0.03:$ & $16.40 \pm 0.07:$ \\
\hline S Ori 16 & $16.578 \pm 0.010:$ & $16.64 \pm 0.03:$ & $16.592 \pm 0.008:$ & $16.62 \pm 0.03$ \\
\hline S Ori J053847.2-025756 & $16.832 \pm 0.015$ & $16.842 \pm 0.015$ & $16.848 \pm 0.009$ & $16.748 \pm 0.020$ \\
\hline S Ori J053829.0-024847 & $16.83 \pm 0.03$ & $16.856 \pm 0.019$ & $16.86 \pm 0.03$ & $16.81 \pm 0.02$ \\
\hline S Ori J053954.3-023719 & $16.939 \pm 0.015$ & $16.838 \pm 0.008$ & $16.832 \pm 0.008$ & $16.82 \pm 0.03$ \\
\hline S Ori J053825.4-024241 & $16.96 \pm 0.02$ & $17.11 \pm 0.10$ & $16.811 \pm 0.012$ & $16.85 \pm 0.03$ \\
\hline S Ori 25 & $17.063 \pm 0.019$ & $16.915 \pm 0.011$ & $16.989 \pm 0.015$ & $16.932 \pm 0.019$ \\
\hline S Ori J053826.1-024041 & $16.92 \pm 0.04$ & $16.97 \pm 0.04$ & $16.99 \pm 0.03$ & $16.93 \pm 0.03$ \\
\hline S Ori 27 & $17.03 \pm 0.03$ & $17.06 \pm 0.02$ & $17.01 \pm 0.02$ & $17.03 \pm 0.03$ \\
\hline S Ori J053922.2-024552 & $17.056 \pm 0.012$ & $17.06 \pm 0.03$ & $17.077 \pm 0.015$ & $17.050 \pm 0.014$ \\
\hline S Ori J054014.0-023127 & $17.069 \pm 0.016$ & $16.983 \pm 0.016$ & $16.95 \pm 0.03$ & $17.07 \pm 0.03$ \\
\hline S Ori 28 & $17.207 \pm 0.019$ & $17.165 \pm 0.013$ & $17.217 \pm 0.015$ & $17.15 \pm 0.03$ \\
\hline S Ori 31 & $17.207 \pm 0.016$ & $17.230 \pm 0.017$ & $17.252 \pm 0.007$ & $17.226 \pm 0.019$ \\
\hline S Ori 30 & $17.273 \pm 0.016$ & $17.297 \pm 0.016$ & $17.284 \pm 0.010$ & $17.290 \pm 0.010$ \\
\hline S Ori 32 & $17.33 \pm 0.02$ & $17.300 \pm 0.017$ & $17.356 \pm 0.013$ & $17.34 \pm 0.02$ \\
\hline S Ori J054004.5-023642 & $17.579 \pm 0.012$ & $17.535 \pm 0.013$ & $17.506 \pm 0.007$ & $17.516 \pm 0.020$ \\
\hline S Ori 36 & $17.707 \pm 0.009$ & $17.710 \pm 0.009$ & $17.710 \pm 0.004$ & $17.713 \pm 0.008$ \\
\hline S Ori J053918.1-025257 & $18.62 \pm 0.05$ & $18.68 \pm 0.05$ & $18.68 \pm 0.05$ & $18.64 \pm 0.04$ \\
\hline S Ori 42 & $19.064 \pm 0.009$ & $19.142 \pm 0.019$ & $19.088 \pm 0.009$ & $19.04 \pm 0.03$ \\
\hline S Ori 45 & $19.484 \pm 0.013$ & $16.49 \pm 0.03$ & $19.46 \pm 0.03$ & $19.48 \pm 0.03$ \\
\hline S Ori J053929.4-024636 & $19.74 \pm 0.04$ & $19.76 \pm 0.03$ & $19.78 \pm 0.04$ & $19.73 \pm 0.02$ \\
\hline S Ori 71 & $19.91 \pm 0.04$ & $19.96 \pm 0.02$ & $19.901 \pm 0.012$ & $20.02 \pm 0.02$ \\
\hline S Ori J053849.5-024934 & $20.150 \pm 0.017$ & $20.18 \pm 0.04$ & $20.118 \pm 0.017$ & $20.081 \pm 0.019$ \\
\hline S Ori 51 & $20.22 \pm 0.07$ & $20.24 \pm 0.04$ & $20.23 \pm 0.05$ & $20.23 \pm 0.02$ \\
\hline S Ori 50 & $20.54 \pm 0.09$ & $20.53 \pm 0.03$ & $20.60 \pm 0.04$ & $20.48 \pm 0.02$ \\
\hline S Ori 47 & $20.50 \pm 0.03$ & $20.52 \pm 0.04$ & $20.52 \pm 0.06$ & $20.49 \pm 0.03$ \\
\hline S Ori J053944.5-025959 & $20.78 \pm 0.06$ & $20.75 \pm 0.03$ & $20.75 \pm 0.05$ & $20.74 \pm 0.03$ \\
\hline S Ori 53 & $20.95 \pm 0.06$ & $21.01 \pm 0.03$ & $20.91 \pm 0.06$ & $20.96 \pm 0.04$ \\
\hline S Ori J054007.0-023604 & $21.16 \pm 0.06$ & $21.17 \pm 0.07$ & $21.18 \pm 0.08$ & $21.16 \pm 0.07$ \\
\hline S Ori J053956.8-025315 & $21.27 \pm 0.04$ & $21.29 \pm 0.05$ & $21.24 \pm 0.03$ & $21.25 \pm 0.07$ \\
\hline S Ori J053858.6-025228 & $21.91 \pm 0.20$ & $22.23 \pm 0.18$ & $22.3 \pm 0.2:$ & $22.17 \pm 0.11:$ \\
\hline
\end{tabular}

${ }^{a} 00 \# 1$ : first observing night of WFC00; 00\#2: second observing night of WFC00; 00\#3: third observing night of WFC00; 03: observing night of WFC03.

the WFC03 epoch. The amplitude of their light curves is given in Table 5. These objects represent about $7 \%$ of the browndwarf final sample. Because our observations are not equally sensitive to $I$-band long-term variability as they are to mid- and short-term photometric variability, this percentage represents a lower limit to the true frequency of long-term variable young brown dwarfs.

The exact temporal scale of the observed $I$-band long-term variability, which may be between several days and years, is unknown to us. This kind of variability could be associated with the long-term evolution of surface features, such as dust clouds or solar-like spots, or with the presence of an accretion disc or a close interactive companion. In the case of S Ori J053847.2-025756, spectral data are lacking, and its $\mathrm{H} \alpha$ emission is unknown. Given its relatively high effective temperature $(\sim 2800 \mathrm{~K}$, obtained from its $I-J$ colour $)$, the formation of magnetically-driven spots or the presence of an accretion disc may be favoured over dust condensation (Mohanty et al. 2002; Joergens et al. 2003). The brown dwarf S Ori 42 is $\sim 2$ mag fainter and cooler, with an estimated effective temperature at around $2500 \mathrm{~K}$. Dust condensation is expected at these cool temperatures; however, the notably high $\mathrm{H} \alpha$ emission of S Ori 42 (Table 2) supports the scenarios involving mass infalling.

Among our final list of brown dwarfs, S Ori 71 is the strongest $\mathrm{H} \alpha$ emitter (Table 2). This source is a non-variable object according to our criteria. Nevertheless, the three $\varpi_{\text {LT }}$ are quite close to the 0.95 limit, suggesting that it may be a real long-term variable with an amplitude of $\sim 0.13 \mathrm{mag}$. S Ori 71 is quite faint, and relatively large photometric errors have likely prevented us from detecting a clear magnitude variation. The persistent and strong $\mathrm{H} \alpha$ emission of S Ori 71 suggests that, 
Table 4. Long-, mid- and short- term variability parameters of the final sample. See Sect. 4 for the definition of each $\varpi$.

\begin{tabular}{|c|c|c|c|c|c|c|c|c|c|c|}
\hline Name & $\begin{array}{c}\varpi_{\mathrm{LT}} \\
00 \# 1-03\end{array}$ & $\begin{array}{c}\varpi_{\mathrm{LT}} \\
00 \# 2-03\end{array}$ & $\begin{array}{c}\varpi_{\mathrm{LT}} \\
00 \# 3-03\end{array}$ & $\begin{array}{c}\varpi_{\mathrm{MT}} \\
00 \# 1-00 \# 2\end{array}$ & $\begin{array}{c}\varpi_{\mathrm{MT}} \\
00 \# 1-00 \# 3\end{array}$ & $\begin{array}{c}\varpi_{\mathrm{MT}} \\
00 \# 2-00 \# 3\end{array}$ & $\begin{array}{c}\varpi_{\mathrm{ST}} \\
00 \# 1\end{array}$ & $\begin{array}{l}\varpi_{\mathrm{ST}} \\
00 \# 2\end{array}$ & $\begin{array}{l}\varpi_{\mathrm{ST}} \\
00 \# 3\end{array}$ & $\begin{array}{c}\varpi_{\mathrm{ST}} \\
03 \\
03\end{array}$ \\
\hline S Ori J053847.2-025756 & 0.93 & 0.97 & 0.95 & 0.2 & 0.5 & 0.1 & 0.5 & 0.2 & 0.4 & 0.4 \\
\hline S Ori J053829.0-024847 & 0.6 & 0.9 & 0.8 & 0.5 & 0.6 & 0.1 & 0.8 & 0.5 & 0.9 & 0.5 \\
\hline S Ori J053954.3-023719 & 0.6 & 0.5 & 0.4 & 0.0 & 0.1 & 0.0 & 0.5 & 0.1 & 0.3 & 0.8 \\
\hline S Ori J053825.4-024241 & 0.96 & 1.00 & 0.8 & 0.98 & 0.99 & 0.99 & 0.6 & 0.98 & 0.4 & 0.8 \\
\hline S Ori 25 & 0.97 & 0.6 & 0.9 & 0.97 & 0.94 & 0.94 & 0.6 & 0.4 & 0.6 & 0.5 \\
\hline S Ori J053826.1-024041 & 0.4 & 0.8 & 0.8 & 0.8 & 0.91 & 0.6 & 0.94 & 0.95 & 0.9 & 0.6 \\
\hline S Ori 27 & 0.1 & 0.8 & 0.4 & 0.7 & 0.4 & 0.90 & 0.7 & 0.7 & 0.7 & 0.7 \\
\hline S Ori J053922.2-024552 & 0.3 & 0.5 & 0.6 & 0.2 & 0.6 & 0.6 & 0.3 & 0.5 & 0.2 & 0.4 \\
\hline S Ori J054014.0-023127 & 0.2 & 0.98 & 0.97 & 0.96 & 0.98 & 0.9 & 0.4 & 0.5 & 0.9 & 0.8 \\
\hline S Ori 28 & 0.93 & 0.6 & 0.92 & 0.8 & 0.3 & 0.95 & 0.6 & 0.5 & 0.6 & 0.7 \\
\hline S Ori 31 & 0.7 & 0.1 & 0.6 & 0.6 & 0.8 & 0.8 & 0.6 & 0.7 & 0.2 & 0.5 \\
\hline S Ori 30 & 0.6 & 0.2 & 0.2 & 0.6 & 0.3 & 0.6 & 0.5 & 0.6 & 0.3 & 0.2 \\
\hline S Ori 32 & 0.5 & 0.8 & 0.4 & 0.7 & 0.7 & 0.97 & 0.6 & 0.7 & 0.4 & 0.6 \\
\hline S Ori J054004.5-023642 & 0.94 & 0.7 & 0.3 & 0.8 & 0.95 & 0.90 & 0.3 & 0.5 & 0.2 & 0.5 \\
\hline S Ori 36 & 0.2 & 0.1 & 0.1 & 0.1 & 0.2 & 0.0 & 0.2 & 0.3 & 0.0 & 0.1 \\
\hline S Ori J053918.1-025257 & 0.7 & 0.7 & 0.7 & 0.9 & 0.94 & 0.4 & 0.95 & 0.96 & 0.93 & 0.9 \\
\hline S Ori 42 & 0.6 & 0.98 & 0.8 & 0.9 & 0.5 & 0.8 & 0.1 & $0.97^{*}$ & 0.1 & 0.5 \\
\hline S Ori 45 & 0.1 & 0.0 & 0.6 & 0.1 & 0.6 & 0.7 & 0.1 & 0.7 & 0.7 & 0.6 \\
\hline S Ori J053929.4-024636 & 0.3 & 0.6 & 0.8 & 0.3 & 0.7 & 0.6 & 0.7 & 0.7 & 0.7 & 0.3 \\
\hline S Ori 71 & 0.9 & 0.9 & 0.9 & 0.6 & 0.2 & 0.7 & 0.5 & 0.4 & 0.3 & 0.2 \\
\hline S Ori J053849.5-024934 & 0.8 & 0.94 & 0.5 & 0.3 & 0.5 & 0.7 & 0.1 & 0.6 & 0.1 & 0.2 \\
\hline S Ori 51 & 0.1 & 0.2 & 0.0 & 0.2 & 0.1 & 0.1 & 0.8 & 0.8 & 0.5 & 0.2 \\
\hline S Ori 50 & 0.7 & 0.6 & 0.8 & 0.3 & 0.7 & 0.8 & 0.90 & 0.4 & 0.3 & 0.1 \\
\hline S Ori 47 & 0.2 & 0.6 & 0.4 & 0.3 & 0.3 & 0.0 & 0.1 & 0.7 & 0.6 & 0.1 \\
\hline S Ori J053944.5-025959 & 0.4 & 0.1 & 0.1 & 0.4 & 0.4 & 0.1 & 0.6 & 0.4 & 0.4 & 0.1 \\
\hline S Ori 53 & 0.2 & 0.8 & 0.4 & 0.6 & 0.4 & 0.7 & 0.4 & 0.1 & 0.4 & 0.3 \\
\hline S Ori J054007.0-023604 & 0.0 & 0.3 & 0.1 & 0.2 & 0.2 & 0.0 & 0.3 & 0.7 & 0.5 & 0.7 \\
\hline S Ori J053956.8-025315 & 0.2 & 0.4 & 0.1 & 0.3 & 0.3 & 0.3 & 0.1 & 0.2 & 0.2 & 0.5 \\
\hline
\end{tabular}

* The S Ori 42 light curve was affected by a crossing artificial satellite path.

for this particular object, the emission is probably linked to the presence of an accretion disc or to a close interactive companion (Barrado y Navascués et al. 2002).

\subsection{Mid-term variable brown dwarfs}

Six young brown dwarf candidates show significant nightto-night $I$-band variations during the WFC00 run. They display at least one of the three $\varpi_{\mathrm{MT}}$ values larger than 0.95 . The amplitude of their light curves is provided in Table 5. These six mid-term variable sources represent about $21 \%$ of our final sample. Possible causes that lead to mid-term photometric variability are: variable obscuration by circumsubstellar dust, heterogeneous distribution of hot or cool spots that cover a large percentage of the substellar surface, dust condensates in the atmosphere, variation in accretion rate and transits of faint companions in close orbits. We highlight two cases: S Ori J053825.4-024241 and S Ori 25.

$\mathrm{S}$ Ori J053825.4-024241 is the most variable brown dwarf candidate in our final sample. The amplitude of the light curve, as measured from peak-to-peak, is 0.36 mag. As indicated in Sect. 3.2, the 2MASS $K_{\mathrm{s}}$-band data of S Ori J053825.4-024241 show significant near-infrared excess, which may be indicative of the presence of a surrounding disc, from which the central object may be accreting. Hence, the observed $I$-band variability could be related to episodes of mass accretion or to eclipses caused by inner portions of the disc, as those presumably observed in the pre-main-sequence object KH 15D (Hamilton et al. 2001). Herbst et al. (2002) stated that a correlation between near-infrared excesses and large photometric variability in low-mass $\mathrm{T}$ Tauri stars exists. S Ori J053825.4-024241 possibly represents one of the first examples of a similar correlation among young brown dwarfs (see also those presented by Carpenter et al. 2001, in the Chamaeleon I molecular cloud). However, we note that the connection between near-infrared excesses and the existence of disc accretion is not so straight forward.

Optical spectra are also available for S Ori 25. They show strong $\mathrm{H} \alpha$ emission, with a width of more than $100 \mathrm{~km} \mathrm{~s}^{-1}$ at $10 \%$ of peak intensity (see references given in Table 2). The 


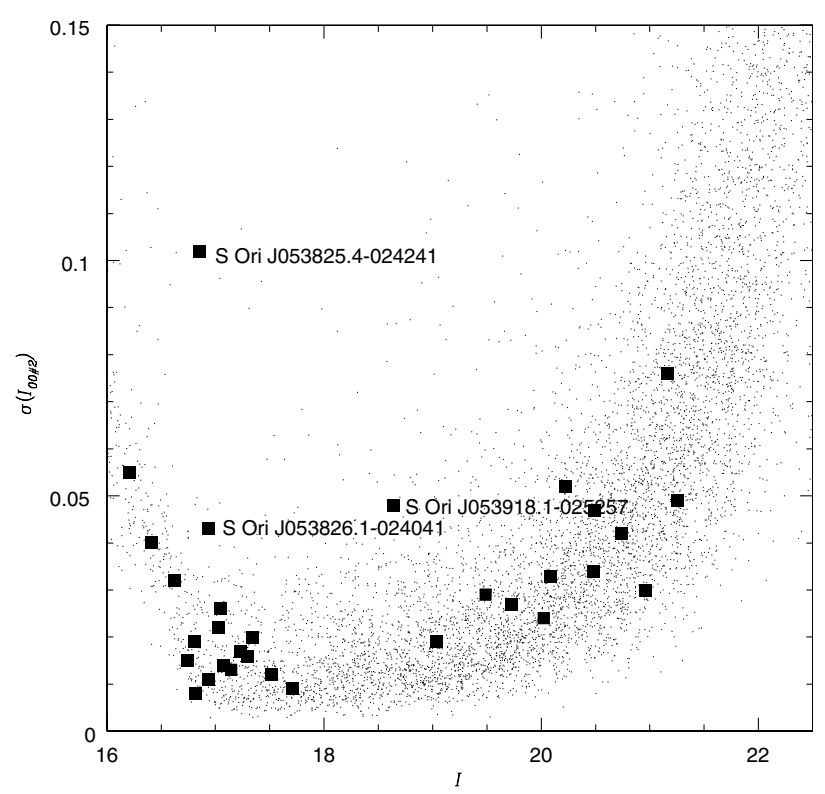

Fig. 7. Standard deviation of the differential light curves versus the I magnitudes for each target (small dots) in the second WFC00 night. Only objects with $\sigma(I)<0.15$ mag are shown. Mean photometric IRAF errors are below 5, 15 and 100 mmag for objects brighter than $I=18$, 20 and 22, respectively. Filled squares denote the brown-dwarf candidates. Short-term variable brown dwarfs (on the basis of the definition of $\left.\varpi_{\mathrm{ST}}\right)$ are labelled.

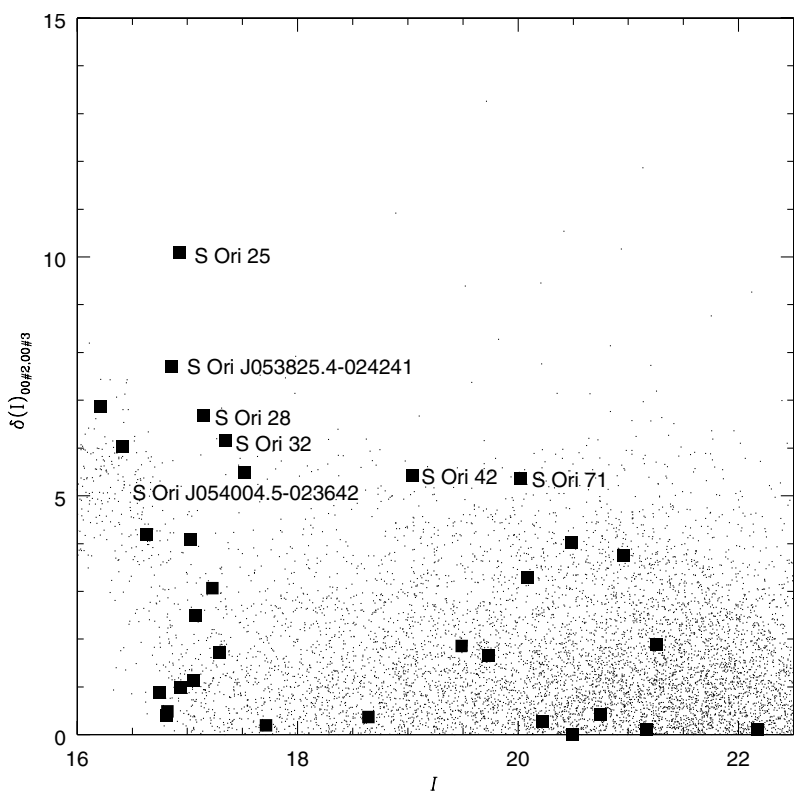

Fig. 8. Dimensionless absolute differences of mean magnitudes between the second and third WFC00 nights vs. apparent $I$ magnitudes. Field objects are plotted as small dots, while filled squares denote the brown-dwarf candidates. Names of several mid-term variable targets are given.

$\mathrm{H} \alpha$ emission of S Ori 25 is remarkably stable on a timescale of years. In addition, this object appears to show a modulation in the light curve with a period of $\sim 40 \mathrm{~h}$, as we will discuss below.

The other four mid-term variable brown dwarf candidates are S Ori J054014.0-023127, S Ori 28, S Ori 32 and S Ori J054004.5-023642. Spectroscopy is not available for

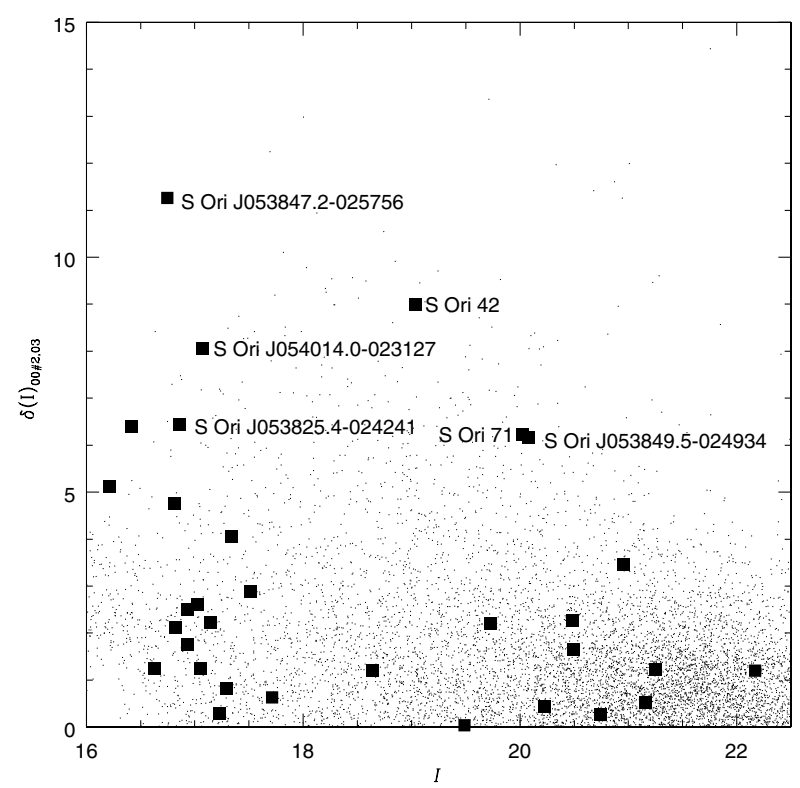

Fig. 9. Dimensionless absolute differences of mean magnitudes between the second WFC00 night and the WFC03 night vs. apparent I magnitudes. Field objects are plotted as small dots, while filled squares denote the brown-dwarf candidates. Names of several variable targets are given.

any of them. However, we note that S Ori J054014.0-023127, for which we determine a photometric I-band amplitude of $\sim 0.12 \mathrm{mag}$, appears quite overluminous in the colour-magnitude diagram of Fig. 2.

\subsection{Short-term variable brown dwarfs}

Three $\sigma$ Orionis brown dwarf candidates are classifed as very likely short-term variables according to our $\varpi_{\mathrm{ST}}$ criterion. They are S Ori J053825.4-024241, S Ori J053826.1-024041 and S Ori J053918.1-025257. Additionally, although the $\varpi_{\mathrm{ST}}$ values of S Ori 27 and S Ori 28 are below the $\varpi_{\text {ST }}=0.95$ boundary, we have measured reliable peaks in the periodograms of their light curves that also suggest classifying them as periodic short-term variable brown dwarfs (see Sect. 5.4). The amplitudes of the light curves are given in Table 5 .

The strong intra-night photometric variations observed in S Ori J053826.1-024041 and S Ori J053918.1-025257 lasts for three or more days. Both sources are apparently stochastic photometric variables with peak-to-peak amplitudes of $\sim 0.10-0.15$ mag on timescales as short as the time interval between consecutive exposures ( $20 \mathrm{~min})$. We cannot classify these two objects as mid-term variables according to our criteria. However, S Ori J053825.4-024241, which has an infrared excess emission at $2.2 \mu \mathrm{m}$, complies with our criteria of mid-term variability.

In principle, short-term variability is more likely to be related to fast variations on the surface of an object than in a disc, where the relevant timescales are likely to be of the order of the revolution period of its particles. However, the rotational modulation scenario requires very fast spin periods, as short as one or two hours. In contrast, intense vertical motions of 
Table 5. Summary of photometric variability.

\begin{tabular}{|c|c|c|c|}
\hline Name & $\begin{array}{c}\text { Variability } \\
\text { type }^{a}\end{array}$ & $\begin{array}{c}\text { Amplitude } \\
\text { [mag] }^{b}\end{array}$ & Properties $^{c}$ \\
\hline S Ori J053847.2-025756 & LT & $0.09 \pm 0.02$ & - \\
\hline S Ori J053829.0-024847 & - & $\leq 0.03$ & - \\
\hline S Ori J053954.3-023719 & - & $\leq 0.02$ & - \\
\hline S Ori J053825.4-024241 & $\mathrm{MT}+\mathrm{ST}$ & $0.36 \pm 0.04$ & nIR \\
\hline S Ori 25 & MT (P) & $0.15 \pm 0.02$ & $\mathrm{H} \alpha$ \\
\hline S Ori J053826.1-024041 & ST & 0.04 & - \\
\hline S Ori 27 & $\mathrm{ST}(\mathrm{P})$ & 0.03 & - \\
\hline S Ori J053922.2-024552 & - & $\leq 0.019$ & - \\
\hline S Ori J054014.0-023127 & MT & $0.12 \pm 0.05$ & - \\
\hline S Ori 28 & $\mathrm{MT}+\mathrm{ST}(\mathrm{P})$ & $0.07 \pm 0.03$ & - \\
\hline S Ori 31 & $\mathrm{ST}\left(\mathrm{P}^{d}\right)$ & $\leq 0.02$ & - \\
\hline S Ori 30 & - & $\leq 0.015$ & - \\
\hline S Ori 32 & MT & $0.06 \pm 0.02$ & - \\
\hline S Ori J054004.5-023642 & MT & $0.073 \pm 0.014$ & - \\
\hline S Ori 36 & - & $\leq 0.009$ & - \\
\hline S Ori J053918.1-025257 & ST & 0.05 & - \\
\hline S Ori 42 & LT & $0.11 \pm 0.03$ & $\mathrm{H} \alpha$ \\
\hline S Ori 45 & $\mathrm{ST}\left(\mathrm{P}^{d}\right)$ & $\leq 0.03$ & $\mathrm{H} \alpha$ \\
\hline S Ori J053929.4-024636 & - & $\leq 0.03$ & - \\
\hline S Ori 71 & - & $\leq 0.06$ & $\mathrm{H} \alpha$ \\
\hline S Ori J053849.5-024934 & - & $\leq 0.05$ & - \\
\hline S Ori 51 & - & $\leq 0.04$ & - \\
\hline S Ori 50 & - & $\leq 0.05$ & - \\
\hline S Ori 47 & - & $\leq 0.04$ & - \\
\hline S Ori J053944.5-025959 & - & $\leq 0.04$ & - \\
\hline S Ori 53 & - & $\leq 0.08$ & - \\
\hline S Ori J054007.0-023604 & - & $\leq 0.07$ & - \\
\hline S Ori J053956.8-025315 & - & $\leq 0.05$ & - \\
\hline
\end{tabular}

${ }^{a}$ Variability type. LT: long-term; MT: mid-term; ST: short-term; P: periodic; -: no detected variability.

${ }^{b}$ Amplitudes of photometric variability: peak-to-peak amplitudes for LT and MT variables and $\sigma(I)$ amplitudes for ST and non-variables. For the latter, the values must be understood as upper limits on variability.

${ }^{c}$ Properties or prominent spectral features. $\mathrm{H} \alpha$ : strong $\mathrm{H} \alpha$ emission; nIR: near-infrared excess.

${ }^{d}$ Periodic according to Bailer-Jones \& Mundt (2001) and/or ZO03.

convective cells (bubbling) caused by turbulent convection could modify the photospheric coverage of dust clouds or dark magnetic spots on very short timescales. This is supported by the greater importance of the internal heat source during the early stages of evolution of substellar objects, which pushes warm material from the interior to the photosphere. Mass infalling from a disc and formation of a hot spot could also explain the sudden high-amplitude brightening (by more than $200 \mathrm{mmag}$ ) of the unsteady variable S Ori J053825.4-024241 during the second WFCO0 night.

\subsection{A search for periodicities}

Time-series analysis has been performed over our final sample of brown dwarfs, i.e. 56 light curves ( 28 brown-dwarf candidates $\times 2$ epochs). We have used PERIOD, a time-series analysis package (developed by Vik Dhillon on La Palma) within the STARLINK software collection, which includes the Lomb-Scargle (Lomb 1976; Scargle 1982) and the CLEAN (Roberts et al. 1987) algorithms. Frequency search limits were the Nyquist critical frequency and the inverse of the maximum temporal coverage. Five iterations and a gain of 0.1 were used during the CLEAN runs. The periodograms of three targets (namely, S Ori 25, S Ori 27 and S Ori 28) show significant peaks, which are at least five times higher than the typical noise of the periodograms of non-variable field objects of similar brightness.

S Ori 25 is a mid-term variable brown dwarf with a light curve that seems to rise and fall in the three-night WFC00 run. The cleaned periodogram in the top box of the Fig. 10 shows a very powerful peak at $40 \pm 8 \mathrm{~h}\left(0.61 \pm 0.13 \mathrm{day}^{-1}\right)$ and its harmonics. The peak also appears in the Lomb-Scargle periodogram. The WFC03 data are not useful for checking this 


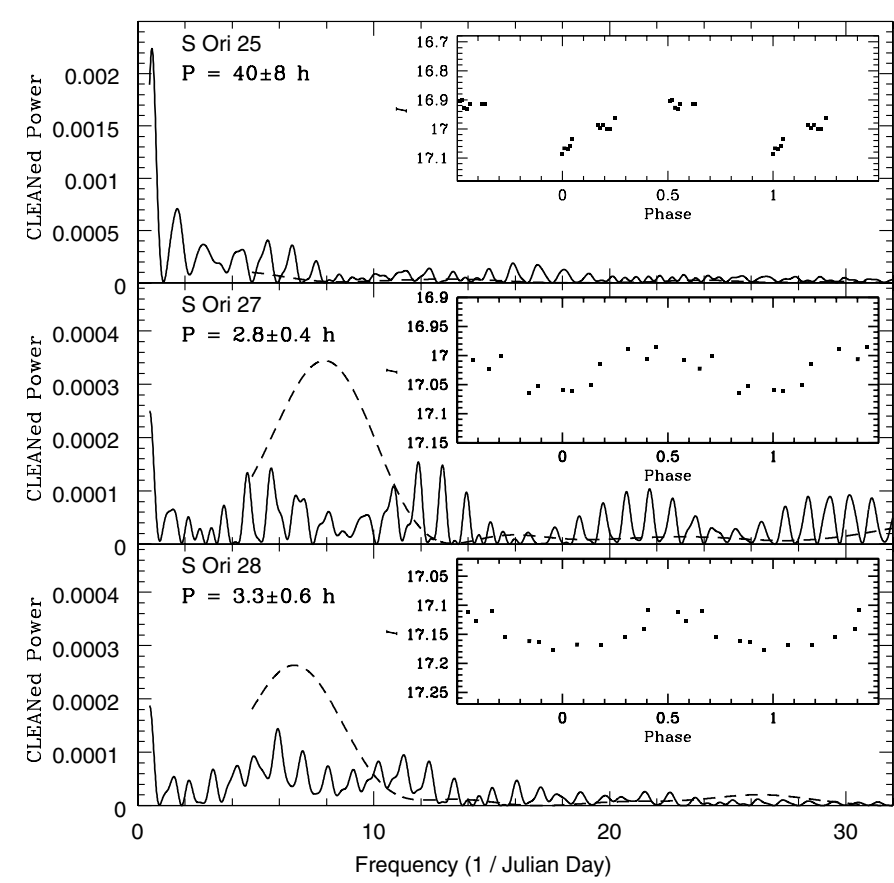

Fig. 10. WFC00 (solid line) and WFC03 (dashed line) light curve periodograms of S Ori $25, \mathrm{~S}$ Ori 27 and S Ori 28 (from top to bottom). Insets show the phased light curves associated with each peak of the periodogram (WFC00, WFC03 and WFC03, respectively). Note that the scale of the cleaned power and $I$ magnitude and the epoch of S Ori 25 are different from S Ori 27 and 28. Further details are given in the text.

detection, as the coverage was only over $\sim 5 \mathrm{~h}$. We will adopt a radius of $0.45 \pm 0.05 R_{\odot}{ }^{4}$ for $\mathrm{S}$ Ori 25 . The uncertainty in the radius determination is estimated from the age interval $1-8 \mathrm{Myr}$ and the evolutionary tracks of Chabrier et al. (2002). The projected rotational velocity, $v \sin i$, of $9.4 \pm 1.0 \mathrm{~km} \mathrm{~s}^{-1}$ (measured by Muzerolle et al. 2003) gives a maximum rotational period of $P / \sin i=58 \pm 9 \mathrm{~h}$ for S Ori 25 , which is compatible with our results. Furthermore, using the measured rotational period, we can constrain both the inclination angle $(i=$ $46_{-13}^{+16}$ degrees) and the rotational velocity $\left(14 \pm 4 \mathrm{~km} \mathrm{~s}^{-1}\right)$. The $40 \mathrm{~h}$ period is greater by far than those in the literature for field brown dwarfs (except for the $238 \mathrm{~h}$ period detected in 2MASSW J1300425+191235 by Gelino et al. 2002), but similar to those recently discovered by Joergens et al. (2003) in the young Chamaeleon I star-forming region. We note that while the duration of the periodic modulation of S Ori 25 appears to be generally longer than that of field brown dwarfs, its $v \sin i$ measurement is similar (Mohanty \& Basri 2003; BailerJones 2004). In addition, the strong $\mathrm{H} \alpha$ emission in S Ori 25 suggests mass accretion. A surrounding accretion disc rotationally locked to the young central object could explain the low angular velocity of S Ori 25 . The angular velocity would be kept roughly constant at a low rate until the disc dissipates and the locking brakes. A similar picture is thought to occur in classical T Tauri stars.

The periodograms of S Ori 27 and S Ori 28 display single peaks at $2.8 \pm 0.4$ and $3.3 \pm 0.6 \mathrm{~h}$, respectively.

\footnotetext{
${ }^{4} 1 R_{\odot}=6.9599 \times 10^{8} \mathrm{~m}$.
}

Folded WFC03 light curves clearly show sinusoidal modulations with amplitudes of about $30 \mathrm{mmag}$, as illustrated in Fig. 10. Unfortunately, the WFC03 data cover roughly one cycle; hence, the determined periods are quite uncertain. The WFC00 light curves do not show periodical variations in time scales of $\sim 3 \mathrm{~h}$. We recall that $\mathrm{S}$ Ori 28 is also a mid-term variable brown dwarf candidate.

This kind of variability, possibly related to surface rotation, could be caused by heterogeneities spread over the photosphere (dust or spots) that evolve over timescales of several hours. The rapid evolution of atmospheric features that lead to the detection of "false" periods if only pieces of light curves are taken into account has been previously noticed by Bailer-Jones \& Mundt (2001) and Gelino et al. (2002). Analogously to the Type II $p$ classical T Tauri stars described by Herbst et al. (1994), hot spots linked to unsteady accretion or rotation could originate the modulations. Hot spots do not last for hundreds or thousands of rotations, as cool spots do.

Other studies of photometric variability of $\sigma$ Orionis cluster substellar members have been published by Bailer-Jones \& Mundt (2001) and ZO03. S Ori 31 and S Ori 45 are the only objects in common with this work. Bailer-Jones \& Mundt (2001) claimed a rotation period of $7.5 \pm 0.6 \mathrm{~h}$ for $\mathrm{S}$ Ori 31. Although this timescale is suitably studied with the WFC00 data, the suggested amplitude of the photometric variation is quite small, $\sim 10 \mathrm{mmag}$. These authors also found a peak of $30 \pm 8 \mathrm{~min}$ in the periodogram of S Ori 45 , a $\sim 25 M_{\text {Jup }}$ M 8.5 brown dwarf with variable, strong $\mathrm{H} \alpha$ emission. A comparably short period ( $46 \mathrm{~min}$ ) has also been claimed by ZO03. However, as noted by those authors, given the expected radius of this young brown dwarf, such a short period cannot be associated with rotation because it would imply an extremely fast rotational velocity inconsistent with hydrostatic equilibrium.

ZO03 also detected a second, more powerful, modulation in their optical and infrared light curves of S Ori 45 with a period of 2.5-3.6 $\mathrm{h}$ that could be linked to rotation. The present WFC00 dataset has already been considered by ZO03. Our new analysis led to a very similar optical light curve. We retrieved the claimed period once the data points around phase 0.5 were excluded, as suggested in ZO03 after using the information from the infrared photometry. According to our rather conservative criteria for the determination of photometric variability, S Ori 45 lies close to the borderline between variable and nonvariable sources. We note that this is also the case of the periodic brown dwarfs S Ori 27 and S Ori 28. From our work and the data collected from the literature, we find that the frequency rate of periodic brown dwarfs in our final sample is around $18 \%$.

\subsection{Final remarks}

Of our final list of twenty eight young brown dwarfs in the $\sigma$ Orionis cluster, nine show some kind of optical photometric variability (short-, mid-, long-term and periodic types) with a $5 \%$ chance of a false positive detection, which represents $39 \%$ of the sample. This frequency rate increases up to $46 \%$ if S Ori 27, S Ori 28 (periodic short-term variables with 


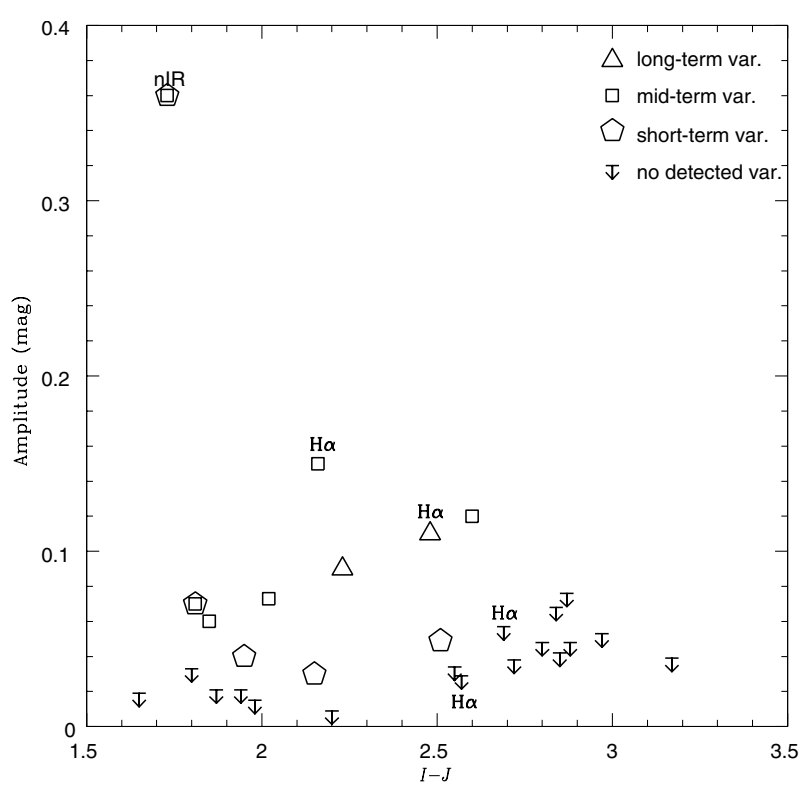

Fig. 11. Amplitudes of variability versus $I-J$ colour. Open triangles, squares and pentagons denote long-, mid- and short-term variables, respectively. Upper limits are shown for the rest of the objects in our sample. Strong $\mathrm{H} \alpha$ emission and near-infrared excess are also indicated.

slightly larger false positive detection probabilities), S Ori 31 and S Ori 45 (periodic brown dwarfs reported in the literature) are also considered. If we only take into account the $\sigma$ Orionis brown dwarf candidates brighter than $I=19.5$, for which the photometric errors are reasonably low, the frequency of variability turns out to be $72 \%$. We summarize our results in Table 5, where the abbreviations LT, MT and ST stand for long-, mid- and short-term variability, respectively. If a periodic signal is reported either in the present study or in the literature, the letter "P" is used. S Ori 27, 28, 31 and 45 are considered as ST variables because of their short photometric periodicities. I-band amplitudes or scatters of the light curves and other interesting properties, like strong $\mathrm{H} \alpha$ emission and near-infrared excess, are also provided in Table 5.

In Fig. 11 we plot maximum photometric amplitudes for the final sample of objects against the $I-J$ colour, which is an indicator of temperature and mass. We use different symbols for the various types of variability, and downward-point arrows for sources with no detected variability. As inferred from the figure, there is no clear trend between amplitudes and the colour of the brown dwarfs. S Ori J053825.4-024241 shows the largest amplitude, which, as discussed above, may be associated with the presence of a surrounding disc. It is also noteworthy from the figure that the largest $I$-band variabilities are found in the group of mid- and long-term variable brown dwarfs. We find $I$-band photometric variability in $\sigma$ Orionis cluster members with masses in the range 70-25 $M_{\mathrm{Jup}}$.

\section{Conclusions and summary}

We have photometrically monitored in the $I$-band a sample of thirty two young brown-dwarf candidates in the young star cluster $\sigma$ Orionis. Many of them are spectroscopically confirmed as bona fide $\sigma$ Orionis substellar members. They are $\sim 3$ Myr old, and their masses span from the substellar limit down to the planetary-mass borderline. Twenty-eight of these substellar objects have reliable variability indicators, and their light curves have been analysed in detail. This is one of the largest samples of young brown dwarfs ever monitored for photometric variability.

With a $5 \%$ chance of a false positive detection, we have found that nine of our targets show I-band variability on various timescales. If we include the periodic young brown dwarfs S Ori 27 and S Ori 28 found in our work, and S Ori 31 and $\mathrm{S}$ Ori 45 reported in the literature, the incidence of variable $\sigma$ Orionis brown dwarfs is around $46 \%$. Such a frequency is higher than that (about 33\%) found among very low mass field stars and brown dwarfs. The measured amplitudes of variability are also more than three times larger in this study. This different behaviour can be ascribed to the youth of the $\sigma$ Orionis brown dwarfs. We classify photometric variability on the basis of long-, mid- and short-term variations.

- Long-term photometric variability (year-to-year variations). Two substellar candidates (a minimum of $7 \%$ of the final sample) show variations in the mean differential magnitudes measured at two epochs 2.09 years apart, with amplitudes of $0.09-0.11 \mathrm{mag}$. They show neither inter- nor intra-night variations.

- Mid-term variability (day-to-day variations). Six targets (21\%) appear to be variable from night to night, with amplitudes of variation in the range 0.06-0.36 mag. Three of them have magnitude variations greater than $0.1 \mathrm{mag}$, which may make these the brown dwarfs with the largest amplitudes detected to date. S Ori 25 shows a $\sim 40 \mathrm{~h}$ periodic modulation in the light curve, consistent with the recent determination of the $v \sin i$ parameter, which yields a rotational velocity of $14 \pm 4 \mathrm{~km} \mathrm{~s}^{-1}$. Magnetic braking due to an accretion disc could account for the long period.

- Short-term variability (scale of hours). Three of our objects apparently display random non-periodic photometric $I$-band variability on timescales of less than a few hours. The amplitudes of the light curves of two of these objects appear to be roughly steady during at least three consecutive nights with standard deviations at the 0.04-0.05 mag level. The third short-term variable brown dwarf, S Ori J053825.4-024241, is also a mid-term variable and shows the largest variability in our final sample. With slightly larger false positive detection probabilities, S Ori 27 and S Ori 28 display peaks in the WFC03 periodograms at around $3 \mathrm{~h}$, which could be related to fast rotation periods, such as those found in other young brown dwarfs and evolved ultracool field dwarfs. These rapid rotations could be explained by the dissipation of a surrounding disc, or by the evolution of surface features on timescales of a few rotations.

We note the correlation found between large amplitude of variability and the detection of a near-infrared excess or strong $\mathrm{H} \alpha$ emission, which could be an evidence of a substellar accretion disc. Four out of thirteen brown dwarfs with available spectra show strong $\mathrm{H} \alpha$ emission (i.e. $p E W(\mathrm{H} \alpha)>30 \AA$ ). Two 
of them (S Ori 25 and S Ori 42) are variable with amplitudes of $\sim 0.12$ mag and with timescales longer than one day. Regarding the other two, S Ori 45 has been independently confirmed as as photometrically variable by ZO03, and S Ori 71 is suspected to be a faint long-term variable. An infrared excess in the $K_{\mathrm{s}}$ band has been detected in the largest-amplitude variable brown dwarf of the sample, S Ori J053825.4-024241. This is one of the first substellar objects sharing a correlation between near-infrared excess and large photometric variability, in analogy to low-mass T Tauri-like stars.

A single variability scenario can hardly explain simultaneously all the temporal scales involved. The presence of surrounding accretion discs, heterogeneous coverage over the photosphere by cool magnetic spots or fast-evolving dust clouds, mass transfer from or eclipses due to a companion have been suggested as possible causes of the variability. Both long-term, multi-band coverage monitoring and complete spectroscopic studies are needed to conclude unambiguously which is the preferred variability scenario in young objects below the substellar limit.

Acknowledgements. We thank C. A. L. Bailer-Jones for a careful reading of the manuscript and for his suggestions, which have clearly improved this manuscript. We would like to thank to E. Martín and D. Barrado y Navascués for helpful comments, and Terry Mahoney for revising the English of the manuscript. Based on observations made with the Isaac Newton Telescope (INT) operated on the island of La Palma by the Isaac Newton Group in the Spanish Observatorio del Roque de Los Muchachos of the Instituto de Astrofísica de Canarias. Partial financial support was provided by the Spanish Ministerio de Ciencia y Tecnología proyect AYA2001-1657 of the Plan Nacional de Astronomía y Astrofísica. Part of this work has been supported by the Spanish Programa Ramón y Cajal. This research has made use of the SIMBAD database, operated at CDS, Strasbourg, France. This publication makes use of data products from the Two Micron All Sky Survey, which is a joint project of the University of Massachusetts and the Infrared Processing and Analysis Center/California Institute of Technology, funded by the National Aeronautics and Space Administration and the National Science Foundation.

\section{References}

Bailer-Jones, C. A. L., \& Mundt, R. 1999, A\&A, 348, 800

Bailer-Jones, C. A. L., \& Mundt, R. 2001, A\&A, 367, 218

Bailer-Jones, C. A. L. 2002, A\&A, 389, 963

Bailer-Jones, C. A. L., \& Lamm, M. 2003, AJ, 339, 477

Bailer-Jones, C. A. L. 2004, 419, 703

Baraffe, I., Chabrier, G., Allard, F., \& Hauschildt, P. H. 2002, A\&A, 382,563

Baraffe, I., Chabrier, G., Allard, F., \& Hauschildt, P. H. 2003, Brown Dwarfs, held 20-24 May 2002 at University of Hawai'i, Honolulu, Hawai'i, ed. E. Martín (San Francisco: Astronomical Society of the Pacific), Proc. of IAU Symp., \#211, 41

Barrado y Navascués, D., Zapatero Osorio, M. R., Béjar, V. J. S., et al. 2001, A\&A, 377, L9

Barrado y Navascués, D., Zapatero Osorio, M. R., Martín, E. L., et al. 2002, A\&A, 393, L85

Barrado y Navascués, D., Béjar, V. J. S., Mundt, R., et al. 2003, A\&A, 404, 171

Barrado y Navascués, D., \& Martín, E. L. 2003, AJ, 126, 2997

Barrado y Navascués, D., Mohanty, S., \& Jayawardhana, R. 2004, ApJ, accepted
Béjar, V. J. S., Zapatero Osorio, M. R., \& Rebolo, R. 1999, ApJ, 521, 671

Béjar, V. J. S., Martín, E. L., Zapatero Osorio, M. R., et al. 2001, ApJ, 556,830

Béjar, V. J. S., Zapatero Osorio, M. R., \& Rebolo, R. 2004, Astron. Nachr., in press

Brown, A. G. A., de Geus, E. J., \& de Zeeuw, P. T. 1994, A\&A, 289, 101

Burgasser, A. J., Kirkpatrick, J. D., Reid, I. N., et al. 2000, AJ, 120, 473

Burgasser, A. J., Liebert, J., Kirkpatrick, J. D., \& Gizis, J. E. 2002, AJ, 123, 2744

Carpenter, J. M., Hillenbrand, L. A., \& Skrutskie, M. F. 2001, AJ, 121, 3160

Carpenter, J. M., Hillenbrand, L. A., Skrutskie, M. F., \& Meyer, M. R. 2002, AJ, 124, 1001

Chabrier, G., Baraffe, I., Allard, F., \& Hauschildt, P. 2000, ApJ, 542, 464

Clarke, F. J., Tinney, C. G., \& Covey, K. R. 2002a, MNRAS, 332, 361

Clarke, F. J., Oppenheimer, B. R., \& Tinney, C. G. 2002b, MNRAS, 335,1158

Clarke, F. J., Tinney, C. G., \& Hodgkin, S. T. 2003, MNRAS, 341, 239

Comerón, F., Fernández, M., Baraffe, I., Neuhäuser, R., \& Kaas, A. A. 2003, A\&A, 406, 1001

Cutri, R. M., Skrutskie, M. F., van Dyk, S., et al. 2003, VizieR Online Data Catalog: II/246, Originally published in: University of Massachusetts and Infrared Processing and Analysis Center, (IPAC/California Institute of Technology)

de Zeeuw, P. T., Hoogerwerf, R., de Bruijne, J. H. J., Brown, A. G. A., \& Blaauw, A. 1999, AJ, 117, 354

Enoch, M. L., Brown, M. E., \& Burgasser, A. J. 2003, AJ, 126, 1006

Fedorovich, V. P. 1960, Perem. Zvezdy, 13, 166

Fernández, M., \& Comerón, F. 2001, A\&A, 380, 264

Gelino, C. R., Marley, M., Holtzman, J. A., Ackerman, A. S., \& Lodders, K. 2002, ApJ, 577, 433

Hall, P. B. 2002, ApJ, 564, L89

Hamilton, C. M., Herbst, W., Shih, C., \& Ferro, A. J. 2001, ApJ, 554, L201

Herbst, W., Herbst, D. K., Grossman, E. J., \& Weinstein, D. 1994, AJ, 108, 1906

Herbst, W., Rhode, K. L., Hillenbrand, L. A., \& Curran, G. 2000, AJ, 119,261

Herbst, W., Bailer-Jones, C. A. L., Mundt, R., Meisenheimer, K., \& Wackermann, R. 2002, A\&A, 396, 513

Jayawardhana, R., Mohanty, S., \& Basri, G. 2002, ApJ, 578, L141

Jayawardhana, R., Mohanty, S., \& Basri, G. 2003, ApJ, 592, 282

Joergens, V., Fernández, M., Carpenter, J. M., \& Neuhäuser, R. 2003, ApJ, 594, 971

Königl, A. 1991, ApJ, 370, L39

Lamm, M. H., Bailer-Jones, C. A. L., Mundt, R., Herbst, W., \& Scholz, A. 2004, 417, 557

Landoldt, A. U. 1992, AJ, 104, 372

Landstreet, J. D., \& Borra, E. F. 1978, ApJ, 224, L5

Lee, T. A. 1968, ApJ, 152, 913

Lomb, N. R. 1976, Ap\&SS, 39, 447

Liebert, J., Kirkpatrick, J. D., Cruz, K. L., et al. 2003, AJ, 125, 343

Martín, E. L., \& Zapatero Osorio, M. R. 1997, MNRAS, 286, L17

Martín, E. L., Zapatero Osorio, M. R., \& Lehto, H. 2001, ApJ, 557, 822

Mohanty, S., Basri, G., Shu, F., Allard, F., \& Chabrier, G. 2002, ApJ, 571,469

Mohanty, S., Jayawardhana, R., \& Barrado y Navascués, D. 2003, ApJ, 593, L109 
Mohanty, S., \& Basri, G. 2003, ApJ, 583, 451

Muench, A. A., Alves, J., Lada, C. J., \& Lada, E. A. 2001, ApJ, 558, L51

Muzerolle, J., Hillenbrand, L., Calvet, N., Briceño, C., \& Hartmann, L. 2003, ApJ, 592, 266

Nakajima, T., Tsuji, T., Maihara, T., et al. 2000, PASJ, 52, 87

Natta, A., \& Testi, L. 2001, A\&A, 376, L22

Natta, A., Testi, L., Comerón, F., et al. 2002, A\&A, 393, 597

Oliveira, J. M., Jeffries, R. D., Kenyon, M. J., Thompson, S. A., \& Naylor, T. 2002, A\&A, 382, L22

Oliveira, J. M., Jeffries, R. D., \& van Loon, J. Th. 2004, MNRAS, 347, 1327

Perryman, M. A. C., Lindegren, L., Kovalevsky, J., et al. 1997, A\&A, 323, L49

Persi, P., Marenzi, A. R., Gómez, M., \& Olofsson, G. 2001, A\&A, 376, 907

Rebull, L. M. 2001, AJ, 121, 1676

Roberts, D. H., Lehár, J., \& Dreher, J. W. 1987, AJ, 93, 968
Saumon, D., Hubbard, W. B., Burrows, A., et al. 1996, ApJ, 460, 993

Scargle, J. D. 1982, ApJ, 263, 835

Stetson, P. B. 1996, PASP, 108, 851

Terndrup, D. M., Krishnamurthi, A., Pinsonneault, M. H., \& Stauffer, J. R. 1999, AJ, 118, 1814

Testi, L., Natta, A., Oliva, E., et al. 2002, ApJ, 571, L155

Tinney, C. G., \& Tolley, A. J. 1999, MNRAS, 304, 119

Wilking, B. A., Greene, T. P., \& Meyer, M. R. 1999, AJ, 117, 469

Zapatero Osorio, M. R., Béjar, V. J. S., Rebolo, R., Martín, E. L., \& Basri, G. 1999, 524, L115

Zapatero Osorio, M. R., Béjar, V. J. S., Pavlenko, Ya., et al. 2002a, A\&A, 384, 937

Zapatero Osorio, M. R., Béjar, V. J. S., Martín, E. L., Barrado y Navascués, D., \& Rebolo, R. 2002b, ApJ, 569, L99

Zapatero Osorio, M. R., Caballero, J. A., Béjar, V. J. S., \& Rebolo, R. 2003, A\&A, 408, 663 (ZO03) 